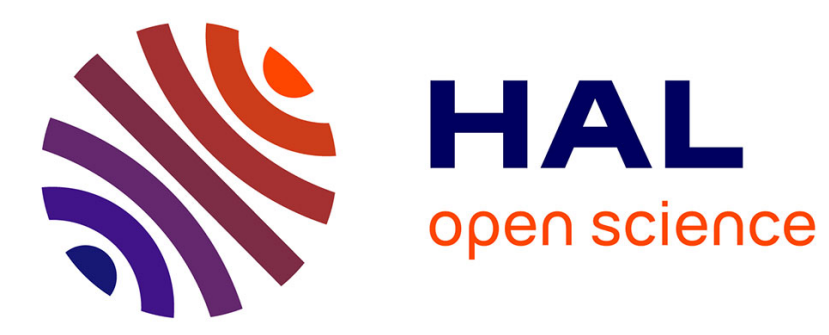

\title{
Formal measures for semantic interoperability assessment in cooperative enterprise information systems
}

\author{
Esma Yahia, Alexis Aubry, Hervé Panetto
}

\section{To cite this version:}

Esma Yahia, Alexis Aubry, Hervé Panetto. Formal measures for semantic interoperability assessment in cooperative enterprise information systems. Computers in Industry, 2012, 63 (5), pp.443-457. 10.1016/j.compind.2012.01.010 . hal-00662878

\section{HAL Id: hal-00662878 \\ https://hal.science/hal-00662878}

Submitted on 25 Jan 2012

HAL is a multi-disciplinary open access archive for the deposit and dissemination of scientific research documents, whether they are published or not. The documents may come from teaching and research institutions in France or abroad, or from public or private research centers.
L'archive ouverte pluridisciplinaire HAL, est destinée au dépôt et à la diffusion de documents scientifiques de niveau recherche, publiés ou non, émanant des établissements d'enseignement et de recherche français ou étrangers, des laboratoires publics ou privés. 


\title{
Formal measures for semantic interoperability assessment in
}

\section{cooperative enterprise information systems}

\author{
Esma Yahia*, Alexis Aubry, Hervé Panetto
}

\author{
Research Centre for Automatic Control (CRAN), University of Lorraine, CNRS, \\ Campus Scientifique, Faculté des Sciences et Technologies, BP 70239, 54506 Vandoeuvre-lès-Nancy Cedex, France \\ \{Esma.Yahia, Alexis.Aubry, Herve.Panetto\}@cran.uhp-nancy.fr
}

\begin{abstract}
The growing complexity of modern organisations poses a series of challenges, among them the cooperation between autonomous and heterogeneous information systems in distributed networks. Actually, Information Systems $(I S)$ are said to be cooperative if they share common goals in their environment and jointly contribute to achieve these common goals. Obviously, this presupposes the ability to exchange information and then to use it in accordance with each information system required. In the literature, these features refer to interoperability. In this context, one of the main issues concerns the evaluation of the lack of interoperability between Cooperative Information Systems (CIS) through the measurement of their semantic gaps. In order to achieve this purpose, this paper proposes a mathematical formalisation of the semantic relationships between CIS conceptual models. The resulting formal model is then analysed for evaluating the lack of interoperability implications to the global information systems shared goals. The proposed approach is illustrated through a case study dealing with a B2M (Business to Manufacturing) interoperability requirement between an Enterprise Resource Planning (ERP) system and a Manufacturing Execution System (MES) application
\end{abstract}

Keywords: Cooperative Information Systems, interoperability assessment, semantic gap, fact-oriented, manufacturing information systems, formal measures.

\section{Introduction}

As cooperative environment progressively evolve, two (or more) Information Systems (IS) need to interoperate between each other in order to fulfil a given objective determined by the cooperation context. Typically, this cooperation requires that heterogeneous $I S$ s coordinate with each other by sharing information. The interoperability occurs when each one of those $I S \mathrm{~s}$ is able to successfully use the exchanged information. When it is related to exchange and use of information, or to carry out an operation on behalf of another system, this is commonly called interoperation [1]. Interoperation is also characterised by Fisher, in [2], as the relationship of the exchange and the cooperative use of information. 
In practice, the achievement of the cooperation depends strongly on the effectiveness of the interoperation between the participating systems. That means that, at least, those systems must be minimally interoperable with each other to complete the global system goal. In this context, assessing the consequence of the lack of interoperability between systems is a critical issue.

The intent of this paper is to focus on the assessment of interoperability assessment by defining some formal metrics which quantify and qualify the level of interoperability between heterogeneous systems. This will allow, at the end, putting in place the necessary actions in order to improve the interoperation process.

Section 2 presents the interoperability issues in the context of Cooperative Information Systems $(C I S)$ and the kinds of barriers that prevent the interoperability achievement. Among these barriers, the semantic barrier is the most critical when considering the comprehension of the exchanged information. It then concerns the evaluation of semantic gaps when information is exchanged between the related CISs.

To determine those CIS semantic gaps, section 3 postulates that it is necessary to conceptualise the knowledge embedded in each CIS and this, using methods that allow emphasizing the semantics of these CIS conceptual models.

Section 4 is devoted to the formalisation of the semantic relationships between conceptual models entities.

The resulting formalisation is then used, in section 5 , to evaluate the consequences of the lack of interoperability in the effectiveness of the Cooperative Information Systems through the formalisation of two correlated measures, $v$ and $\varepsilon$, respectively the potential interoperability and the interoperability effectiveness.

In order to illustrate the proposed approach, we detail a case study in the section 6 . This case study deals with B2M (Business to Manufacturing) interoperability requirements between an Enterprise Resource Planning (ERP) and a Manufacturing Execution System (MES) applications that can be seen as CISs.

Finally, we conclude this paper with some remarks and perspectives for further works.

\section{Interoperation assessment in CISs}

For achieving a given goal, CISs work together and develop different interactions or interoperability relationships between each other. They are autonomous with regards to their own mission and they are heterogeneous with regards to their architecture and content.

Among the different definitions for interoperability, we quote the most significant ones in our context. Interoperability is defined in [3] as the ability of two or more systems or elements to exchange information and to use the information that has been exchanged. Carney has extended, in [4], the above definition by adding the notion of purpose related to the goal of interoperation and the notion of context (the environment in which the entities exist). ). Interoperability is then defined as the 
ability of a collection of communicating entities to (i) share specified information and (ii) operate on that information according to a shared operational semantics (iii) in order to achieve a specified purpose in a given context. Moreover, Carney, in [4], consider that the essence of interoperation is that it is a relationship between systems in a cooperative context and secondly, that a minimum of communication is needed to ensure the interoperation. Panetto, in [5], complements this definition stating that interoperable systems are by necessity compatible, but the converse is not necessarily true. To realize the power of networking through robust information exchange, one must go beyond compatibility. In fact to interoperate one system must provide a service that is used by another. This cannot be achieved without, at a minimum, communication from the provider to the consumer of the service [4].

When depicting interoperation among information systems, we focus on the relationship between CISs and not the manner of communication.

To achieve interoperability between CISs, various points of view or barriers must be taken into account at technical, conceptual and organisational levels ([6], [7], [8]).

The software engineering community has already developed mature technologies and standards (e.g. XML, SOA, WS ...) to remove the technical barriers. Removing the organisational barriers is certainly the most difficult issue as it deals with people and organisations. Even if it is a critical problem for CISs, it is out of scope of this paper.

The main contribution of this paper concerns then, analysing and measuring the semantic gap occurring when CISs exchange information by facilitating the formalisation of concepts mapping between CIS internal models [9].

Current research on interoperability measurement mainly focuses on maturity measures based on a variety of models. We can quote LISI (Levels of Information System Interoperability) [10], OIM (Organizational Interoperability Model) [11], LCIM (Levels of Conceptual Interoperability Model) [12] and the new standard ISO 11354-1:2010 [13]. Those approaches rely on qualitative but subjective assessments based on general evaluation criteria as quoted in [5], [14] and [15]. Moreover, these approaches lack formal definition of their criteria and need a high level of expertise to be applied in an industrial context $[16]$.

Some studies have tried to explore quantitative measures ( [16], [17]) for describing interoperability relationships, but considering the participating systems as black boxes without any concerns about the details and the semantics of the exchanged information. The approach proposed by [18] and [19] provides a framework for expressing and evaluating quantitatively the semantic relationships between multiple information systems. Those information systems are seen as a collection of processes that produce services to accomplish a well-defined objective. Thus, evaluating the interoperability of theses information systems is based on the evaluation of the degree of coupling between processes. This evaluation is allowed through evaluating the semantic relationships and identifying the activity replication and overlapping, as well as the aspects related to interaction/cooperation. 
Some significant studies have shown the usefulness of the measures precision and recall that originate from Information Retrieval (IR) [20] and have been adapted for Ontology Matching Quality (OMQ) in [21] and [22]. Those measures are mainly based on the comparison of an expected result and the effective result of the evaluated system, for instance, the set of semantic relationships to be retrieved by ontology matching tools. We could not apply those measures to evaluate interoperability between information systems as they are only useful to evaluate the ability of the ontology matching tools for retrieving the right semantic relationships between information systems. Thus precision and recall do not measure the ability of two information systems to interoperate, which is the goal of this paper.

In order to contribute to the interoperability evaluation issue, we propose an approach based on the formalisation of the semantic relationships between systems (seen as white boxes) by analysing the detailed semantics of their conceptual model and relationships. We are then discussing the lack of interoperability evaluation between those systems by measuring semantic gaps through the formalised semantic relationships and this by identifying potential and effective interoperability metrics.

\section{Fact-oriented perspective for semantics modelling}

\subsection{Fact-oriented modelling}

While studying an implemented information system model, we observe that its semantics is tacit as it is scrambled due to implementation requirements. Interoperability assessment has then to take place at a conceptual level in order to enact any "grain" of semantics embedded in the application, allowing bringing out the tacit semantics and making them explicit [23]. Indeed, the starting point of our approach consists as a very detailed conceptual model where each "atom" of semantics embedded in any artefact (classes, attributes, associations, constraints ...) is presented with a unified concrete syntax.

However, one main difficulty in operating with the various conceptual models comes out from the fact that the majority of those models have been made by different experts with various modelling experiences. That can lead, for instance, to many conceptual representations for the same semantics. Since the majority of conceptual models have been fulfilled a posteriori and not a priori, implementation-based functionalities and constraints can cause interferences in the semantics understanding of those models. Let us consider, for instance, two concepts extracted from two different conceptual models, in Figure 1. Intuitively, those concepts (represented as UML classes) carry out similar semantics (at least from a global point of view), but are modelled differently. For instance, the WEIGHT of a PRODUCT on the right side of the figure is represented by a single class due to, for example, an implementation constraint. When other classes are related to this class, a query for specific values related to the weight is enabled. In contrast, on the left side of the figure, the WEIGHT of a PRODUCT is modelled by two attributes (its value and its unit). 


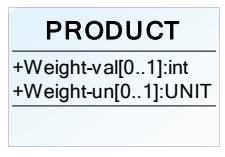

$I_{1}$

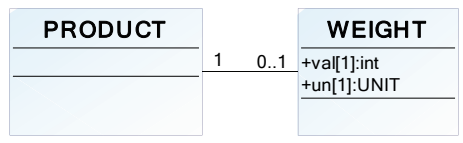

$\mathrm{IS}_{2}$

Figure 1. Two extracts of conceptual models.

In order to cope with such heterogeneous modelling patterns, we propose to transform those conceptual models by normalizing them to a fine-grained semantic model based on a fact-oriented modelling approach [24].

Fact-oriented modelling is a conceptual, natural language based approach avoiding such conflicting conceptual representations. It queries the information semantics of business domains in terms of the underlying facts of interest, where all facts and rules may be verbalised in a language readily understandable by users of those business domains [24]. Fact-oriented models are attribute-free, treating all elementary facts as relationships.

Object-Role Modelling (ORM) is the most popular fact-oriented approach. In fact, ORM makes no explicit use of attributes; instead it pictures the world in terms of lexical concepts (LC) and non-lexical concepts (NLC) that play roles (take part in relationships) [25]. A lexical concept models information that has an associated value. It could have a data type (string or integer for example). Besides, the non-lexical concept models information that is composed by other information.

ORM leads to a greater semantics stability and populatability, as well as facilitates natural verbalisation [24].

When applying the fact-oriented modelling on the examples of the Figure 1 using ORM 1 notation, we obtain the following models (Erreur ! Source du renvoi introuvable.) that eases the semantics enactment.
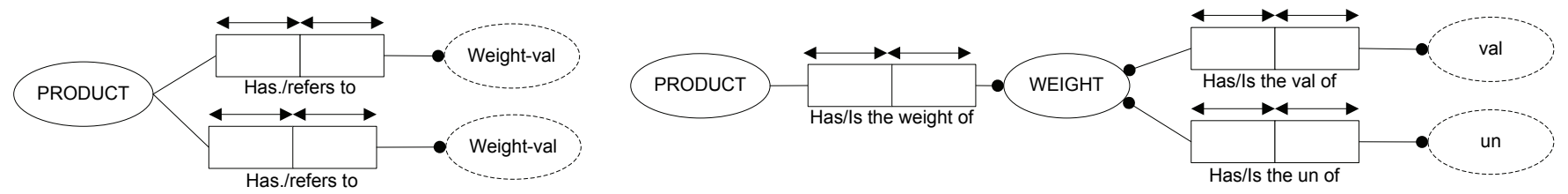

Figure 2. The conceptual models of Figure 1 transformed by a fact-oriented modelling perspective using the ORM 1 notation

In our work, we could use ORM as a modelling language. However, existing conceptual models, in an industrial context, are mainly represented with the UML notation. Hence getting an attribute-free conceptualisation could be made using the UML notation but based on the ORM approach. Taking into account the ORM definitions, we will use the UML class diagram notation and we then call the UML concepts and the UML attributes as respectively non-lexical concepts and lexical concepts, in reference to those ORM ones. In Figure 3, we presented the conceptual models of Figure 1 transformed by a fact-oriented modelling perspective based on UML notation. 


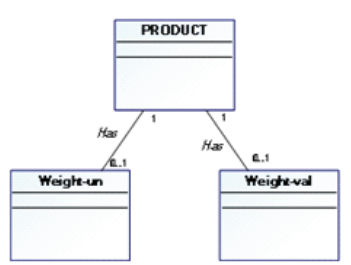

$\mathrm{IS}_{1}$

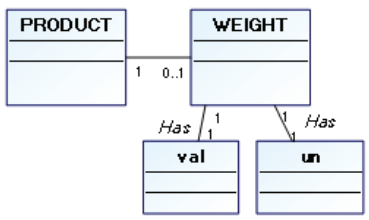

$\mathrm{IS}_{2}$

Figure 3. The conceptual models of Figure 1 transformed by a fact-oriented modelling perspective based on UML notation In conclusion, all the pre-existing conceptual models are then normalised by applying such modelling patterns based on a factoriented modelling approach with an UML notation. This allows us to make explicit the underlying implicit semantics.

\subsection{Core and extended semantics}

When considering an available fact-oriented conceptual model from one $I S$, we can distinguish the mandatory concepts from the non-mandatory ones through the identification of the mandatory associations (associations with constrained) among those concepts.

In fact, the set of mandatory concepts reflects all the necessary semantics that ensure coherency and comprehensibility of the studied conceptual model. It comprises all the non-lexical and lexical concepts linked to constrained association roles with a multiplicity equal to 1 or $1 .{ }^{*}$.

On the opposite, the non-mandatory concepts correspond to the non-constrained roles (multiplicity equal to $0 . .1$ or $*$ ) and are only enriching the semantics of those $I S$ conceptual models.

Somehow, the set of mandatory concepts corresponds to the core semantics that is embedded into a given $I S$ conceptual model: without this semantics, the $I S$ cannot operate. It thus represents the essence of the $I S$ that is the core semantics explicitly embedded into the conceptual model. The extended semantics includes then the mandatory and the non-mandatory concepts.

\subsection{Some mathematical definitions}

We define, for each $I S$ conceptual model, the following notations.

Definition 1. $C_{I S}$ is the set of the identified lexical and non-lexical concepts, formally defined by

$$
C_{I S}=\left\{c_{i} \mid c_{i} \text { is a lexical or non - lexical concept from the } I S \text { conceptual model }\right\}
$$


We define moreover two subsets as follows:

- $\quad N L C_{I S}$ is the subset of $C_{I S}$ restricted to the non-lexical concepts and,

- $L C_{I S}$ is the subset of $C_{I S}$ restricted to the lexical concepts.

We can note that:

$$
\begin{gathered}
C_{I S}=N L C_{I S} \cup L C_{I S} \\
N L C_{I S} \cap L C_{I S}=\emptyset
\end{gathered}
$$

Definition 2. $\operatorname{Rel}_{I S}$ is the set of the identified binary relationships between concepts. Formally, it is defined by

$$
\operatorname{Rel}_{I S}=\left\{\operatorname{rel}\left(c_{i}, c_{j}\right) \mid\left(c_{i}, c_{j}\right) \in C_{I S} \times C_{I S} \wedge c_{i} \text { is associated with } c_{j}\right\}
$$

Definition 3. $\operatorname{Mult}\left(\operatorname{rel}\left(c_{i}, c_{j}\right)\right)$ represents the multiplicity of the role of $c_{j}$ in a given association between $c_{i}$ and $c_{j}$ from the if it exists.

For each $\left(c_{i}, c_{j}\right) \in\left(C_{I S}\right)^{2}$, if $\operatorname{rel}\left(c_{i}, c_{j}\right)$ exists then we have $\operatorname{Mult}\left(\operatorname{rel}\left(c_{i}, c_{j}\right)\right) \in\{*, 0 . .1,1,1 . . *\}$ and it is read $c_{j}$ is associated to $c_{i}$ with a multiplicity equal to $\operatorname{Mult}\left(\operatorname{rel}\left(c_{i}, c_{j}\right)\right)$.

Definition 4. $M C_{I S}$ is the subset of $\mathrm{C}_{\mathrm{IS}}$ restricted to mandatory concepts (the core semantics). It is formally defined by

$$
M C_{I S}=\left\{c_{i} \mid \exists\left(c_{i}, \operatorname{rel}\left(c_{i}, c_{j}\right)\right) \in C_{I S} \times \operatorname{Rel}_{I S} \wedge \operatorname{Mult}\left(\operatorname{rel}\left(c_{i}, c_{j}\right)\right) \in\{1,1 . . *\}\right\}
$$

We define moreover two subsets as follows:

- $M N L C_{I S}$ is the subset of $C_{I S}$ restricted to the mandatory non-lexical concepts and,

- $M L C_{I S}$ is the subset of $C_{I S}$ restricted to the mandatory lexical concepts.

We can note that:

$$
\begin{gathered}
M C_{I S}=M N L C_{I S} \cup M L C_{I S} \\
M N L C_{I S} \cap M L C_{I S}=\emptyset \\
M N L C_{I S}=M C_{I S} \cap N L C_{I S} \\
M L C_{I S}=M C_{I S} \cap L C_{I S}
\end{gathered}
$$




\section{Semantic relationships formalisation}

\subsection{The proposed formalisation approach}

The specified scenario that is considered in this paper consists in two information systems $\left(I S_{1}\right.$ and $\left.I S_{2}\right)$ that are interoperating in the context of cooperative enterprises. We are then considering their respective fact-oriented conceptual models (section 3.1) in order to assess their potential and effective interoperability (section 5). Conforming to the interoperability definition given in section 2, we could establish that $I S_{1}$ interoperates with $I S_{2}$ if the following properties are satisfied (Figure 4):

P1. $I S_{1}$ is able to communicate some information with $I S_{2}$, and

P2. $I S_{2}$ is able to understand at least partially the semantics of the information exchanged, and

P3. $I S_{2}$ operates on that exchanged information.

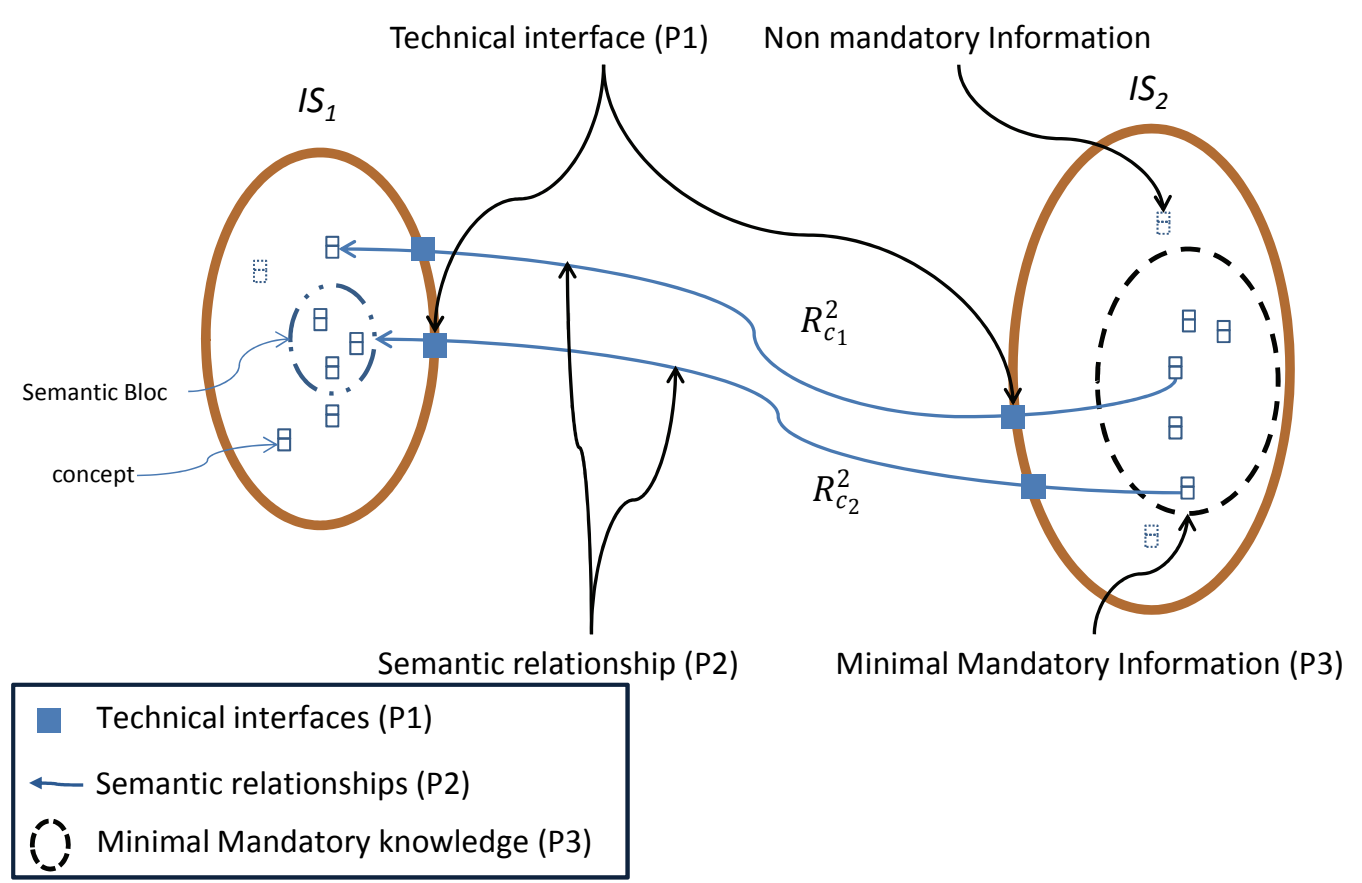

Figure 4. Interoperability properties

Firstly, to exchange information, $I S_{I}$ and $I S_{2}$ must have compatible interfaces (P1, technical interoperability). Secondly, to study the property P2, we need to identify the different semantic relationships between the fact-oriented conceptual models. Finally, $I S_{2}$ makes effective the usage of the exchanged information (i.e. operates P3) if this information is enough to comply with $I S_{2}{ }^{\prime}$ minimal mandatory information (core semantics defined in section 3.2).

Formally, P2 and P3 are thus satisfied if:

$$
\forall c_{i}^{2} \in M C_{I S_{2}}, \exists\left(c_{q_{1}}^{1}, \ldots, c_{q_{p}}^{1}\right) \in\left(C_{I S_{1}}\right)^{p} \mid \operatorname{sem}\left(c_{i}^{2}\right) \subseteq \operatorname{sem}\left(c_{q_{1}}^{1}, \ldots, c_{q_{p}}^{1}\right)
$$

Where $\operatorname{sem}\left(c_{i}^{2}\right)$ is the semantics of $c_{i}^{2}$. 
That means that a semantic relationship $R_{c_{i}}^{2}$ (formally defined as $R_{c_{i}}^{2}=\left\langle c_{i}^{2},\left(c_{q_{1}}^{1}, \ldots, c_{q_{p}}^{1}\right)\right\rangle$ ) can be identified and $R_{c_{i}}^{2}=\left\langle c_{i}^{2},\left(c_{q_{1}}^{1}, \ldots, c_{q_{p}}^{1}\right)\right\rangle$ can be read as: $c_{i}^{2}$ is semantically subsumed by $\left(c_{q_{1}}^{1}, \ldots, c_{q_{p}}^{1}\right)$.

The reason for considering only the mandatory concepts from $M C_{I S_{2}}$ (the core semantics) is due to the fact that non-mandatory concepts are, by definition, not necessary for the application implementing $C_{I S_{2}}$ to operate correctly.

$R_{c_{i}}^{2}$, if it exists, is identified by a domain expert who is the only guarantor of the relevance of the resulting relationships. To achieve this, the expert can take profits from existing approaches (schema matching and morphism, ontology mapping). The goal of this paper is not to develop such an approach. For this reason, a state of the art on these approaches is presented in section 4.2 .

Based on the semantics relationships, identified by the expert, we can define a set of the retrieved semantic relationships denoted as $R_{c}^{2}$ and formally defined as:

$$
R_{c}^{2}=\left\{R_{c_{i}}^{2}=\left\langle c_{i}^{2},\left(c_{q_{1}}^{1}, \ldots, c_{q_{p}}^{1}\right)\right\rangle \mid c_{i}^{2} \in M C_{I S_{2}} \wedge\left(c_{q_{1}}^{1}, \ldots, c_{q_{p}}^{1}\right) \in P\left(C_{I S_{1}}\right)\right\}
$$

where $P\left(C_{I S_{1}}\right)$ is derived from the powerset of $C_{I S_{1}}: P\left(C_{I S_{1}}\right)$ is the set of all $p$-tuples $\left(c_{q_{1}}^{1}, \ldots, c_{q_{p}}^{1}\right)$ that can be made using the elements of $C_{I S_{1}}$, with $p \in\left[1, s_{1}\right]$ and $s_{1}=\left|C_{I S_{1}}\right|$.

We can note that $R_{c}^{2}$ is also the graph of a binary relation $\mathcal{R}^{2}$ such that $\mathcal{R}^{2} \subseteq M C_{I S_{2}} \times P\left(C_{I S_{1}}\right)$.

Considering $c_{i}^{2} \in M C_{I S_{2}}$ and $\left(c_{q_{1}}^{1}, \ldots, c_{q_{p}}^{1}\right) \in\left(C_{I S_{1}}\right)^{P}, c_{i}^{2} \mathcal{R}^{2}\left(c_{q_{1}}^{1}, \ldots, c_{q_{p}}^{1}\right)$ means that a semantic relationship $R_{c_{i}}^{2}$ has been identified by the expert.

We also define $R_{c}^{2 e}$, that is a subset of $R_{c}^{2}$, restricted to the relationships $R_{c_{i}}^{2}=\left\langle c_{i}^{2},\left(c_{q_{1}}^{1}, \ldots, c_{q_{p}}^{1}\right)\right\rangle$ where $\left(c_{q_{1}}^{1}, \ldots, c_{q_{p}}^{1}\right)$ contains only mandatory concepts. Thus, $R_{c}^{2 e}$ can be formally denoted as:

$$
R_{c}^{2 e}=\left\{R_{c_{i}}^{2}=\left\langle c_{i}^{2},\left(c_{q_{1}}^{1}, \ldots, c_{q_{p}}^{1}\right)\right\rangle \mid c_{i}^{2} \in M C_{I S_{2}} \wedge\left(c_{q_{1}}^{1}, \ldots, c_{q_{p}}^{1}\right) \in \mathrm{P}\left(M C_{I S_{1}}\right)\right\}
$$

Two cases can then be considered, for interpreting the semantic relationship $R_{c_{i}}^{2}$ :

i. If there exists at least one $c_{i}^{2} \in M C_{I S_{2}}$ such that the semantic relationship $R_{c_{i}}^{2}$ cannot be identified, there exists a semantic loss when $I S_{1}$ interoperates with $I S_{2}$.

ii. If there exists a semantic relationship $R_{c_{i}}^{2}$ between $c_{i}^{2}$ and a unique or several concepts coming from $C_{I S_{1}}$. There is no semantic loss concerning $c_{i}^{2}$.

Formally, semantic loss occurs during the interoperation process when the following condition is satisfied:

$$
\exists c_{i}^{2} \in M C_{I S_{2}} \mid \forall\left(c_{q_{1}}^{1}, \ldots, c_{q_{p}}^{1}\right) \in \mathrm{P}\left(C_{I S_{1}}\right), \neg c_{i}^{2} \mathcal{R}^{2}\left(c_{q_{1}}^{1}, \ldots, c_{q_{p}}^{1}\right)
$$

When studying the interoperation from $I S_{2}$ to $I S_{1}$, we have to define $R_{c}^{1}$ and $\mathcal{R}^{1}$ following the same syntax as for $R_{c}^{2}$ and $\mathcal{R}^{2}$. 
From these definitions, the main contribution of this paper is then to formally evaluate this semantic loss by analysing the semantic relationships and then measuring the potential interoperability and the interoperability effectiveness in section 5 .

\subsection{Semantic relationships identification approaches}

As presented previously, to derive the semantic interoperability between two CISs, a domain expert must, at first, identify the different existing relationships (i.e. $R_{c}^{1}$ and $R_{c}^{2}$ ) between the various studied conceptual model entities. These semantic relationships define the correspondences between concepts from both CISs by indicating for a given concept from one CIS if it is semantically equivalent to one or more other concepts from the second one. Thus, those relationships correspond to the semantic associations and can be based on intuitive notions such as connectivity and semantic similarity [26].

According to [27], semantics « involves those relations that are meaningful to our IS and can be inferred either based on the data itself or with the help of additional knowledge. To determine the relevance of semantic associations it is necessary to capture the context within which they are going to be interpreted (or the domains of the user interest) ».

We based our work on the hypothesis that the information systems semantics and the semantic relationships retrieved are only guaranteed by the systems experts. We present in this part, the different approaches facilitating the identification of those semantic relationships by the experts. The evaluation of their work to retrieve those relationships could be conducted using precision and recall ([20], [21] and [22]).

Among the existing approaches for addressing those semantic associations, we can cite ontology mapping [28], schema matching [29], [30] and [31] and schema morphism [32]. These approaches typically compute similarities by relating entities via semantic relationships, with, sometimes, a qualitative measure. There exist several possible configurations [33]. In fact, entities could be a simple or complex construction of entities, the mappings could be direct or non-direct [34], The type of mapping relationships could be sorted from manually to semi automatically and automatically mapping and they could range from vaguely to formally specified. A measure assigned to the mapping could denote either a mapping probability or an objectively measurable similarity degree or relatedness [35] that are in the range of [0,1] such as the Cosine similarity measure, Jaccard similarity coefficient, and the Market Basket Model [36].

Both schema matching and schema morphism are mainly non-logical based approaches and require syntactic manipulations [37]. Besides, schema morphism has the advantage to allow some semantic consideration to be realised like the concatenation (for instance, "full-name" is mapped with ("first-name" and "last-name")), and to allow the correctness of information translation to be formally verified [38]. On the contrary, ontology mapping is typically a logic-based approach and involves different ontologies in order to capture the domain-specific semantics of any concept. Those mappings are usually performed 
manually by domain experts who are familiar with one or more specific-industry domain, or accomplished via different techniques based on the comparison of the ontology structures or the linguistic semantic of the concepts [39].

Besides, we could profit from using one existing top domain ontology to specify the context and then to address those semantic associations like in [40]. Indeed, one domain expert must be able to semantically map concepts from one $I S$ to a top domain ontology. For such a top domain ontology, we can cite, for instance, DOLCE [41] and CYC [42] as general cases or, PRONTO [43], ONTO-PDM [44] product ontology for the manufacturing domain.

The choice of a given approach depends on the domain expert's considerations that concern the cost, the time, the efforts and the required expertise to define the semantic relationships. Besides the domain experts are, for sure, the only guarantor of the relevance of the resulting mapping.

\section{Semantic Interoperability Evaluation}

To contribute to the lack of interoperability evaluation between CISS, we adopt the above approach. The definition of the various semantic relationships $R_{c}^{1}$ and $R_{c}^{2}$ (formalised in section 4.1) between conceptual models allows us to determine different measures in order to quantify the CISs semantic gap. Mainly, five major semantic gap configurations could be defined as shown on the Venn Diagrams [45] in Figure 5. When defining the semantic relationships, we are able to determine in which configuration case the studied CISs lie.

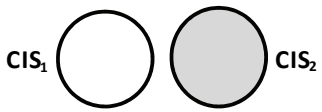

Disjoint

$\mathrm{ClS}_{1} \subseteq \neg \mathrm{ClS}_{2}$

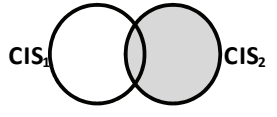

Intersect

$\mathrm{CIS}_{1} \cap \mathrm{CIS}_{2}$

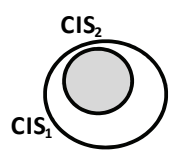

Include

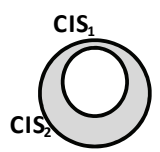

Include

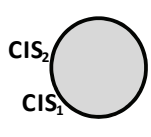

Equal

Figure 5. Semantic gap configurations

\subsection{Semantic Interoperability properties}

According to $\mathrm{He}$, in [46], the "semantic interoperability depends on the system's application domain, and different application domains have different semantic interoperability objectives. In general, it refers to the capability that two software modules or systems can exchange the data with precise meaning, and the receiving party can accurately translate or convert the information carried by the data, including the knowledge, i.e., information and knowledge that can be understood, and ultimately produce an effective collaborative results." 
If we consider two heterogeneous information systems $I S_{1}$ and $I S_{2}$, saying that $I S_{1}$ is semantically interoperable with $I S_{2}$ implies that $I S_{1}$ could exchange information with $I S_{2}$ which operates on it, according to the shared operational semantics, in order to achieve a specified purpose in a given context. Thus $I S_{2}$ is able to use the information acquired from $I S_{1}$ with equivalent meaning to its local data. This definition implies that the set of the semantic relationships between the mandatory concepts from $M C_{I S_{2}}$ and those from $C_{I S_{1}}$ noted as $\boldsymbol{R}_{c}^{2}$ is not empty.

\section{Property 1: Non-symmetry}

The above definition points to the fact that interoperability is not necessarily a symmetric relationship, it could be possible that $I S_{1}$ is interoperable with $I S_{2}$, while $I S_{2}$ is not interoperable with $I S_{1}$. This could be the case in some cooperative information systems, where only one interoperability direction is needed, the other direction is not necessary. In fact, regarding the knowledge (mandatory and non-mandatory) embedded in each CIS (IS 1 and $\left.I S_{2}\right)$, the interoperation could be conducted separately through two ways; from $I S_{1}$ to $I S_{2}$, and/or vice versa.

\section{Property 2: Maximal potential interoperability}

When considering the entire semantics (mandatory and non-mandatory) provided by the concepts from $C_{I S_{1}}$ to define the interoperability relationships $R_{c}^{2}$, we are expecting that all these identified relationships use available concepts, that means that all of their instances are filled by the user (even the non-mandatory concepts from $N M C_{I S_{1}}$ ). That is why we can qualify this interoperability as the maximal potential interoperability. In fact, we could not guaranty that the non-mandatory concepts are completely instantiated by the users of the information system $I S_{1}$ (as they are non-mandatory); this emphasises on the potential of $I S_{1}$ to interoperate with $I S_{2}$.

Definition 1. Maximal Potential Interoperability
Considering two Information Systems $I S_{1}$ and $I S_{2}$, we define the maximal potential interoperability
from $\mathrm{IS}_{1}$ to $\mathrm{IS}_{2}$, the interoperability that is reached when all the concepts of $\mathrm{IS}_{1}$ (even the non-
mandatory ones) are instantiated.

\section{Property 3: Effective interoperability}

When restricting $R_{c}^{2}$ to the mandatory semantics provided by $R_{c}^{2 e}$, we can guaranty that these relationships are effective as the entire semantics provided by $R_{c}^{2 e}$ is by definition available (all the concepts participating to $R_{c}^{2 e}$ are instantiated). Thus, the interoperability which can be deduced from these relationships can be qualified as effective and minimal. In fact, in these relationships, the information is guaranteed to be present (each concept is mandatory and thus instantiated) and understood (the semantic relationship exists). 


\section{Definition 2. Minimal Effective Interoperability}

Considering two Information Systems $I S_{1}$ and $I S_{2}$, we define minimal potential interoperability from $I S_{1}$ to $I S_{2}$, the interoperability that is reached when only the mandatory concepts of $I S_{1}$ are instantiated.

\subsection{Semantic Interoperability levels}

The semantic interoperability can be classified in different levels; full semantic interoperability, partial semantic interoperability and no semantic interoperability. The main features of each level are described below based on [46] and [47]:

\section{i. Full semantic interoperability}

The two information systems can fully share the information and realise the wanted interoperation. For instance $I S_{2}$ is able to use the information acquired from $I S_{1}$ with equivalent meaning to its local information and then this received information can be combined seamlessly with the already existing information in $I S_{2}$ and processed homogeneously.

In our case, $I S_{1}$ is said fully interoperable with $I S_{2}$, implies that $\left|R_{c}^{2}\right|=\left|M C_{I S_{2}}\right|$. That means that the whole mandatory concepts from the $M C_{I S_{2}}$ have semantic relationships with some concepts from $C_{I S_{1}}$. In the same way, saying that $I S_{2}$ is fully interoperable with $I S_{1}$, implies that $\left|R_{C}^{1}\right|=\left|M C_{I S_{1}}\right|$.

\section{Definition 3. Full Semantic Interoperability}

Considering two Information Systems $I S_{1}$ and $I S_{2}$, the semantic interoperability from $I S_{1}$ to $I S_{2}$ is said full if $\left|R_{c}^{2}\right|=\left|M C_{I S_{2}}\right|$.

\section{ii. Partial semantic interoperability}

The partial semantic interoperability between information systems entities refers to partial satisfaction of the semantic agreement [...]. The degree of partial semantic interoperability is normally represented by the percentage of shared [semantics]. 100\% represents the [full] interoperability, and lower percentage means [partial] semantic interoperability [46]. That means that $I S_{1}$ is said partially interoperable with $I S_{2}$ when $\left|R_{c}^{2}\right|<\left|M C_{I S_{2}}\right|$. Moreover, $I S_{2}$ is said partially interoperable with $I S_{1}$ when $\left|R_{c}^{1}\right|<\left|M C_{I S_{1}}\right|$.

\section{Definition 4. Partial Semantic Interoperability}

Considering two Information Systems $I S_{1}$ and $I S_{2}$, the semantic interoperability from $I S_{1}$ to $I S_{2}$ is said partial if $\left|R_{c}^{2}\right|<\left|M C_{I S_{2}}\right|$.

\section{iii. No semantic interoperability}


It refers to the situation where there is no semantic interoperability ( $R_{c}^{2}$ is empty) even if the technical interoperability is effective.

\section{Definition 5. No Semantic Interoperability}

Considering two Information Systems $I S_{1}$ and $I S_{2}$, there is no semantic interoperability from $I S_{1}$ to $I S_{2}$ where $\left|R_{c}^{2}\right|=0$.

\subsection{Interoperability assessment}

In order to evaluate the level of interoperability between $C I S s$, we consider in this section only one way, from $I S_{1}$ to $I S_{2}$ without loss of generality. For this reason we take into consideration the mandatory semantics from $I S_{2}$ in order to find the semantic mappings with the entire IS $S_{1}$ knowledge.

Therefore, the following different cases can be distinguished:

i. $\quad$ The semantic relationships do not exist as $R_{c}^{2}$ is empty $\left(\left|R_{c}^{2}\right|=0\right)$. There is a semantic loss and the semantics of $I S_{1}$ and $I S_{2}$ are completely disjoint. Hence $I S_{1}$ is not interoperable with $I S_{2}$.

ii. The semantic relationships belonging to $R_{c}^{2}$ can be computed for just a part of the concepts from $M C_{I S_{2}}\left(\left|R_{c}^{2}\right|<\right.$ $\left.\left|M C_{I S_{2}}\right|\right)$. Hence $I S_{1}$ is partially interoperable with $I S_{2}$.

Then, we will be able to evaluate the semantic gap by quantifying the missing relationships among concepts.

iii. The semantic relationships belonging to $R_{c}^{2}$ can be computed for each concept from $M C_{I S_{2}}\left(\left|R_{c}^{2}\right|=\left|M C_{I S_{2}}\right|\right)$.

The mandatory semantics of $I S_{2}$ is subsumed by those of $I S_{1}$. Hence $I S_{1}$ is fully interoperable with $I S_{2}$.

In the following table, we summarise the possible cases that could occur when studying the interoperability between two information systems (in the both ways: from $I S_{1}$ to $I S_{2}$ and from $I S_{2}$ to $I S_{1}$ ).). 
Table 1. The different interoperability configurations

\begin{tabular}{|l|l|l|l|}
\hline & & & \\
\hline & & & \\
\hline
\end{tabular}

Using this notation:

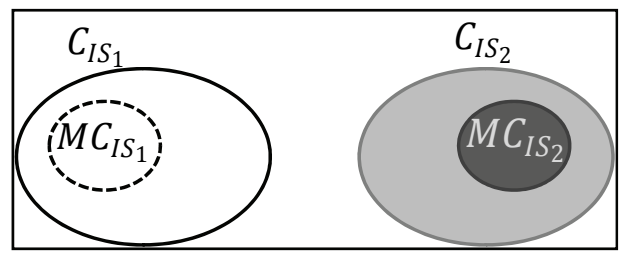

As the full interoperability is seldom achieved, the most significant issue concerns, by consequence, the partial interoperability (when $\left|R_{c}^{1}\right|<\left|M C_{I S_{1}}\right|$ or/and $\left|R_{c}^{2}\right|<\left|M C_{I S_{2}}\right|$ ) as it will provide us a reference scenario to measure the interoperability and to discover the semantic gap and finally the missing semantic relationships. This would be useful, at the end, to bring out all the necessary actions to be taken into account in order to reach a desired level of interoperability.

\subsubsection{Interoperability evaluation}

To evaluate the degree of interoperability between $I S_{1}$ and $I S_{2}$ conceptual models and to characterise the semantic gap, we need to formalise different measures. These measures should be mainly based on the comparison between an expected and a 
real state. The expected state is denoted as $R_{c_{\text {expected }}}^{2}$, and represents the set of the expected semantic relationships needed to ensure a full semantic interoperation $\left(\operatorname{dom} R_{c_{\text {expected }}}^{2}=M C_{I S_{2}},\left|R_{c_{\text {expected }}}^{2}\right|=\left|M C_{I S_{2}}\right|\right)$. The real state corresponds to $R_{c}^{2}$ that is the set of the semantic relationships identified by the expert.

Based on the different semantic interoperability properties identified in section 5.1, we propose two measures to evaluate respectively the potential and the effective interoperability.

\subsubsection{Maximal Potential Interoperability measure}

The potential interoperability measure between two information systems is computed by taking into consideration the maximal potential interoperability property (cf. Section 5.1). Thus, we consider all the retrieved semantic relationships in $R_{c}^{2}$. To measure the potential interoperability, we define $v_{1 \rightarrow 2}(\%)$ as follows:

$$
v_{1 \rightarrow 2}=\frac{\left|R_{c}^{2}\right|}{\left|R_{\text {expected }}^{2}\right|}
$$

$v_{1 \rightarrow 2}$ measures the ratio of those semantic relationships over the total number of the expected semantic relationships to fully interoperate. It then quantifies the maximal potential interoperability. The value $v_{1 \rightarrow 2}$ increases when the semantic gap decreases. The closer to $100 \% v_{1 \rightarrow 2}$ is, the smaller the semantic gap is.

In fact, it is obvious achieving $v_{1 \rightarrow 2}$ equal to $100 \%$ when the expert retrieves the whole set of expected semantic relationships. This corresponds to the full interoperability. If it is less than $100 \%$, it corresponds to a partial interoperability.

The semantic gap is valued by $1-v_{1 \rightarrow 2}$. Moreover, the missing semantics is given by the semantics of the concept $c_{j}^{2}$ from $M C_{I S_{2}}$ for which $R_{c_{j}}^{2}$ cannot be computed: these semantic relationships are identified in $R_{c_{\text {expected }}}^{2} \backslash R_{c}^{2}$.

Therefore, $v_{1 \rightarrow 2}$ alone is not enough to evaluate the interoperability if we consider the effectiveness of the returned semantic relationships. In fact, only the retrieved semantic relationships derived through the mandatory concepts from $M C_{I S_{1}}$ and $M C_{I S_{2}}$ are guaranteed to be effective: this corresponds to $R_{c}^{2 e}$. Thus, we need to measure the effectiveness of the retrieved semantic relationships. Thus, we can formalise an effectiveness measure $\varepsilon_{1 \rightarrow 2}(\%)$ as follows:

$$
\varepsilon_{1 \rightarrow 2}=\frac{\left|R_{c}^{2 e}\right|}{\left|R_{c}^{2}\right|}
$$

The value $\left(1-\varepsilon_{1 \rightarrow 2}\right)$ represents the risk to retrieve non-effective semantic relationships (because it involves nonmandatory concepts).

$v_{1 \rightarrow 2}$ and $\varepsilon_{1 \rightarrow 2}$ as they are defined represent respectively the potential interoperability and the effectiveness measures. 
Typically, $I S_{1}$ may interoperate with $I S_{2}$ with a potential that equals to $v_{1 \rightarrow 2}(\%)$, but with a related effectiveness that equals to $\varepsilon_{1 \rightarrow 2}(\%)$. That means that among the semantic relationships used to compute $R_{c}^{2}$, only $\varepsilon_{1 \rightarrow 2}(\%)$ of them is guaranteed to be effective (without any risk of non-availability of the information).

Besides, if we would like to promote an effective interoperability evaluation, we could consider the interoperation effectiveness property to drive new measures. Indeed, in the following section, we formalise the effective interoperability measure.

\subsubsection{Minimal Effective Interoperability measure}

The effective interoperability evaluation for two information systems could be computed by taking into consideration the minimal necessary amount of semantics that represents the effective mandatory knowledge and that is given through $R_{c}^{2 e}$. This allowed us to compute measures such as $v_{1 \rightarrow 2}^{e}(\%)$ and $\varepsilon_{1 \rightarrow 2}^{e}(\%)$ to evaluate qualitatively the interoperability.

For this, we define:

$$
v_{1 \rightarrow 2}^{e}=\frac{\left|R_{c}^{2 e}\right|}{\left|R_{\text {expected }}^{2}\right|}=\frac{\left|R_{c}^{2}\right|}{\left|R_{\text {expected }}^{2}\right|} \times \frac{\left|R_{c}^{2 e}\right|}{\left|R_{c}^{2}\right|}=v_{1 \rightarrow 2} \times \varepsilon_{1 \rightarrow 2}
$$

$v_{1 \rightarrow 2}^{e}$ measures the ratio of the returned effective semantic relationships over the total number of the expected semantic relationships to fully interoperate.

As we consider all the effective returned semantic relationships, we guarantee the minimal effective interoperability between the studied information systems, but with an effectiveness equal to $100 \%$ as we can also define an associated effectiveness as follows:

$$
\varepsilon_{1 \rightarrow 2}^{e}=\frac{\left|R_{c}^{2 e}\right|}{\left|R_{c}^{2 e}\right|}=100 \%
$$

We can note that $\varepsilon_{1 \rightarrow 2}^{e}$ is always equal to $100 \%$. This is not surprising as $v_{1 \rightarrow 2}^{e}$ measures an effective interoperability.

\subsubsection{Interoperability evaluation synthesis}

In this section, we synthesise the conclusions that can be deduced from the interoperability measures presented above. 
Table 2. Interoperability conclusions following the values of $\left(v_{1 \rightarrow 2}, \varepsilon_{1 \rightarrow 2}\right)$ and $\left(v_{1 \rightarrow 2}^{e}, \varepsilon_{1 \rightarrow 2}^{e}\right)$

\begin{tabular}{|c|c|c|c|}
\hline Type of evaluation & $\begin{array}{l}\text { Interoperability } \\
\text { measure }\end{array}$ & Value & Conclusion \\
\hline \multirow{3}{*}{$\begin{array}{l}\text { Maximal Potential } \\
\text { interoperability }\end{array}$} & \multirow{3}{*}{$v_{1 \rightarrow 2}$} & $=0$ & $\begin{array}{l}I S_{1} \text { is not interoperable with } I S_{2} \text {. The semantic } \\
\text { gap is equal to } 100 \% \text {. }\end{array}$ \\
\hline & & $<100 \%$ & $\begin{array}{l}I S_{1} \text { is partially interoperable with } I S_{2} \text { but only } \\
\varepsilon_{1 \rightarrow 2}(\%) \text { of the semantic relationships given } \\
\qquad \text { by } R_{c}^{2} \text { are effective. } \\
\qquad v_{1 \rightarrow 2} \text { represents the maximal potential } \\
\text { interoperability level that can be reached by } I S_{1} \\
\text { if all the concepts taking part into } R_{c}^{2} \text { would be } \\
\text { mandatory. }\end{array}$ \\
\hline & & $=100 \%$ & $\begin{array}{l}I S_{1} \text { is fully interoperable with } I S_{2} \text { but only } \\
\varepsilon_{1 \rightarrow 2}(\%) \text { of the semantic relationships given } \\
\qquad \text { by } R_{c}^{2} \text { are effective. } \\
\text { This full interoperability could be effective if } \\
\text { all the concepts taking part into } R_{c}^{2} \text { would be } \\
\text { mandatory. }\end{array}$ \\
\hline \multirow{3}{*}{$\begin{array}{l}\text { Minimal Effective } \\
\text { interoperability }\end{array}$} & \multirow{3}{*}{$v_{1 \rightarrow 2}^{e}$} & $=0$ & $\begin{array}{l}I S_{1} \text { is not interoperable with } I S_{2} \text {. The semantic } \\
\text { gap is equal to } 100 \% \text {. }\end{array}$ \\
\hline & & $<100 \%$ & $\begin{array}{l}I S_{1} \text { is partially interoperable with } I S_{2} \text { and this } \\
\text { partial interoperability is effective. } \\
v_{1 \rightarrow 2}^{e} \text { represents the minimal effective } \\
\text { interoperability level that is sure to be reached } \\
\text { by } I S_{1} \text {. }\end{array}$ \\
\hline & & $=100 \%$ & $\begin{array}{l}I S_{1} \text { is fully interoperable with } I S_{2} \text { and this } \\
\text { interoperability is effective. }\end{array}$ \\
\hline
\end{tabular}




\subsubsection{Interoperability measures map}

When evaluating the interoperability (potential and effective), both measures could be computed: an interoperability map can be pictured as in Figure 6. The points $M P I=\left(v_{1 \rightarrow 2}, \varepsilon_{1 \rightarrow 2}\right)$ and $M E I=\left(v_{1 \rightarrow 2}^{e}, \varepsilon_{1 \rightarrow 2}^{e}\right)$ represent respectively the maximal potential interoperability and the minimal effective interoperability.

Starting from these points, the different improvements that could take place can follow two phases:

1. Firstly, reaching the maximal intrinsic interoperability: this corresponds to the point $M I I=\left(v_{1 \rightarrow 2}, \varepsilon_{1 \rightarrow 2}^{e}\right)$ in Figure 6. This effort could be conducted by transforming all the non-mandatory concepts (taking part into $R_{c}^{2}$ ) into mandatory ones.

2. Secondly, reaching the full interoperability (100\% of interoperability with $100 \%$ of effectiveness): this corresponds to a substantial effort as we need to add the missing semantics to reach $(100 \%, 100 \%)$. The missing semantics is identified by the semantics of the concepts taking part into $R_{c_{\text {expected }}}^{2} \backslash R_{c}^{2}$.

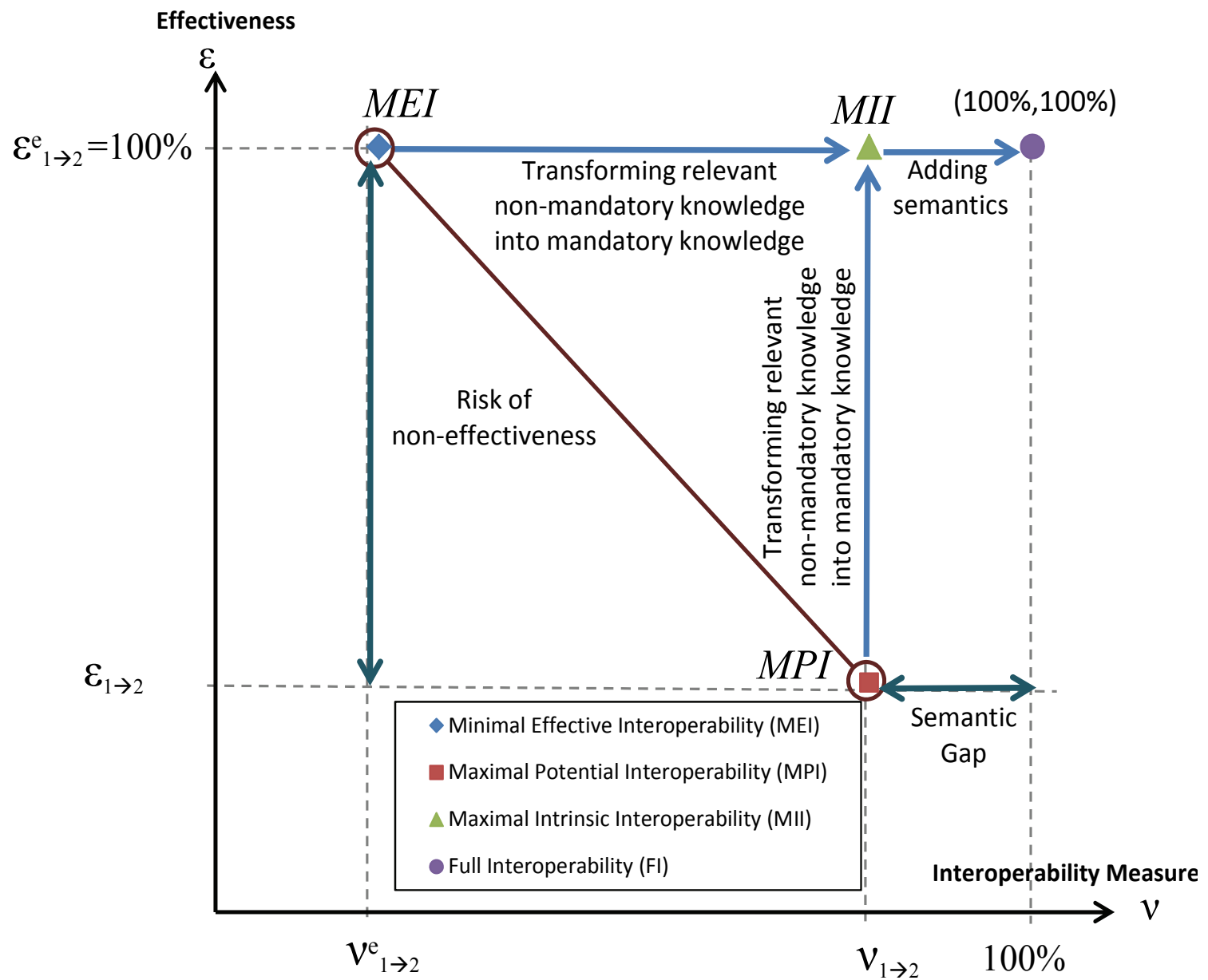

Figure 6. The interoperability measures map 


\section{Case Study: Raw Material Purchase}

In order to illustrate the proposed formalisation approach, we choose the following case study that consists of two information systems dealing with B2M interoperability requirement. These ISs have been provided by a local technical centre: the AIPL-PRIMECA ${ }^{1}$ (Atelier Inter-établissements de Productique Lorrain) in which the ERP Sage X3 application is cooperating with the MES Flexnet application in order to ensure the manufacturing of a certain family of products. In such industrial large scale Enterprise Information Systems, applications comprise a multitude of tables and relations. Flexnet (a MES application) has around 800 tables with 300 relations. Once we conceptualise its model, we get about 600 concepts and 500 associations. SAGE X3 has around 1600 tables with 900 relations, and when it is conceptualised, 1200 concepts and 1000 associations can be highlighted.

Actually, a specified process has been chosen to support our research; it consists of the Raw Material Purchase. We defined both dedicated conceptual models dealing with the concerned collaborative processes. For instance, Figure 7, represents the conceptual model for the purchase order process related to Flexnet.

When considering the long term planning, the ERP calculates, for a given period, its needs in term of materials and then launches some purchase orders. Hence, those purchase orders are exported from ERP to the MES and the MES has to bring forward to the ERP with the stock status and the purchase order status.

Once we apply the fact-oriented modelling using the UML notation, we obtain the normalised conceptual models of Flexnet (Figure 8) and the conceptual model of Sage X3 (Figure 9). The automatic generation of those models is elaborated with the MEGA² tool that provides us with a script editor in Visual Basic.

\footnotetext{
${ }^{1}$ AIPL-PRIMECA, www.aip-primeca.net/lorraine/

${ }^{2} \mathrm{http}: / / \mathrm{www} . \mathrm{mega.com} / \mathrm{en} / \mathrm{c} /$ product/p/product-overview
} 


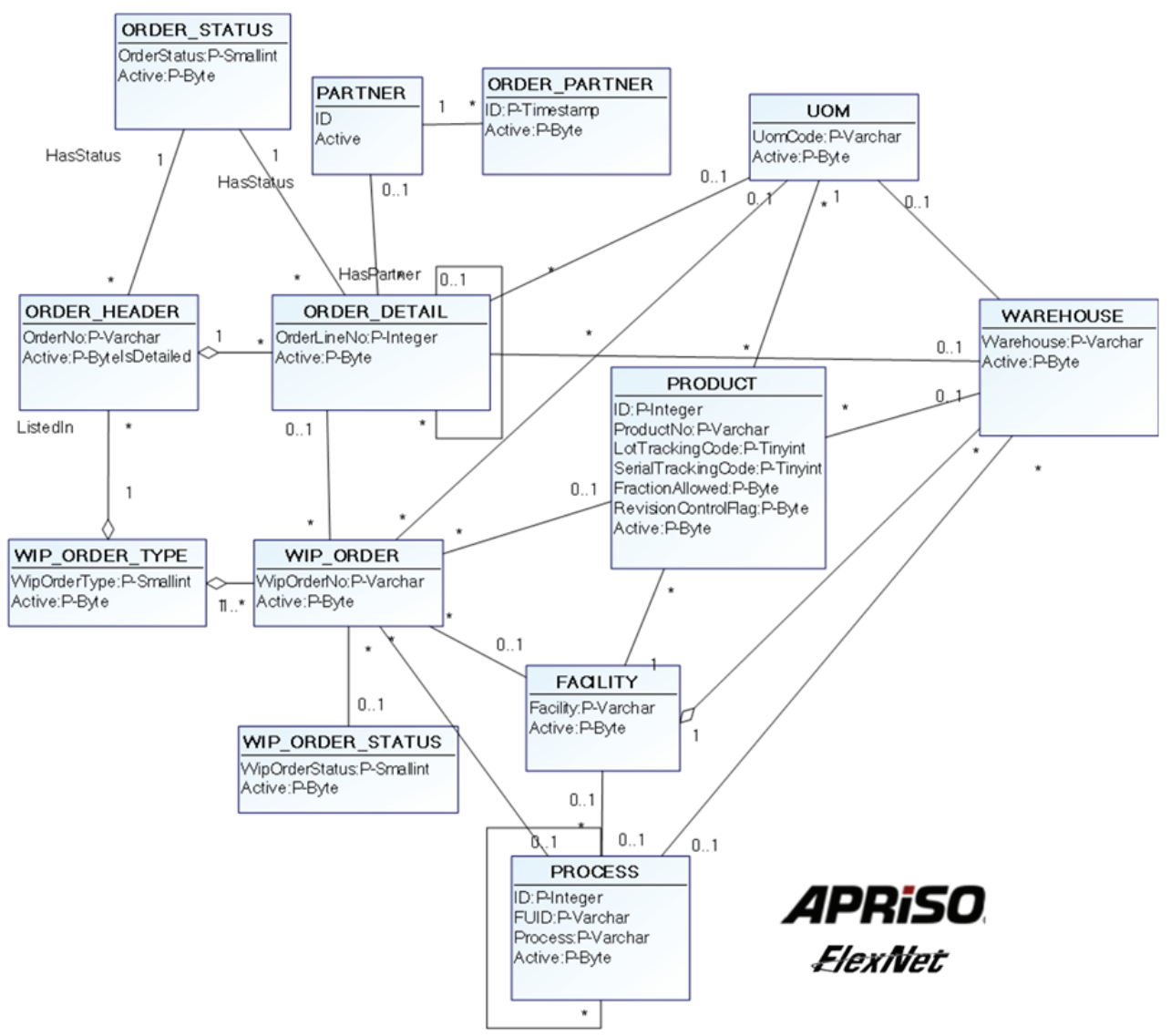

Figure 7. Conceptual model for the purchase order process from Flexnet

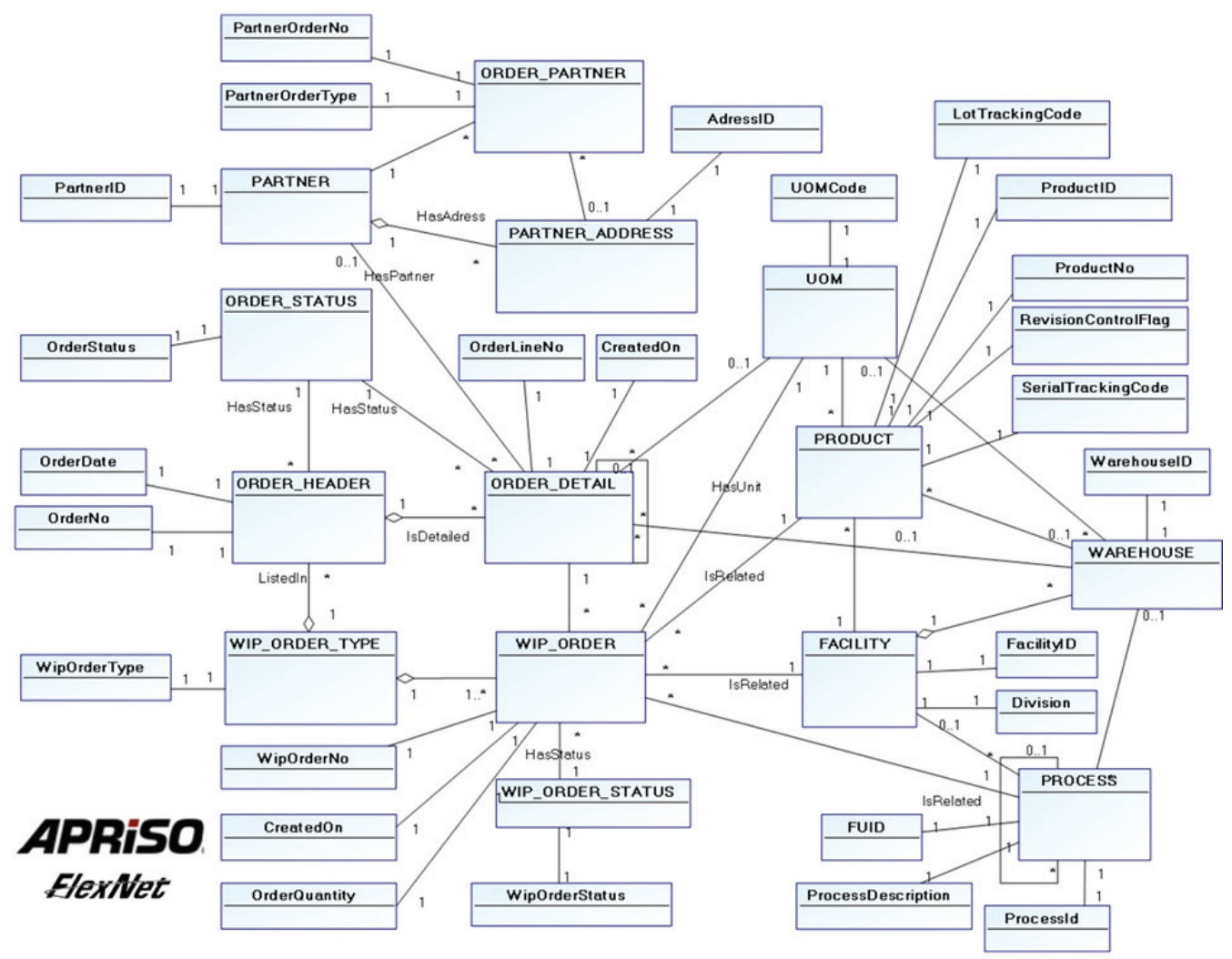

Figure 8. The conceptual model of a purchase order in Flexnet application: fact-oriented model 


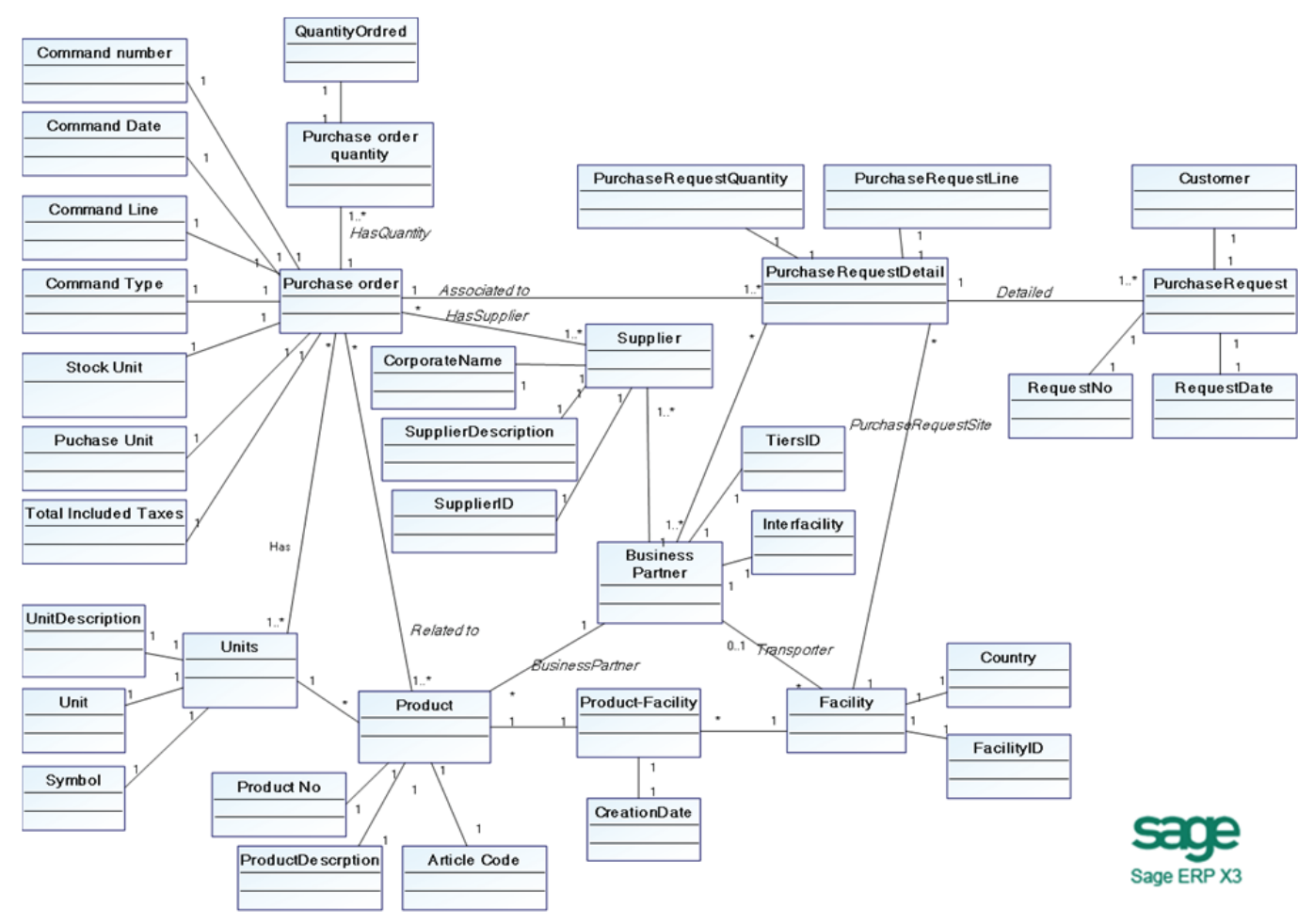

Figure 9. The conceptual model of a purchase order in SAGE X3 application: fact-oriented model

In the following section, we will compute the interoperability measures in order to quantify and qualify both potential and effective interoperation conducted from the MES $\left(I S_{1}\right)$ to the ERP $\left(I S_{2}\right)$.

The same method could be applied to evaluate the semantic interoperability from the ERP to the MES.

6.1. Interoperability evaluation from $\operatorname{MES}\left(I S_{1}\right)$ to the $\operatorname{ERP}\left(I S_{2}\right)$

The domain expert returns the semantic relationships $R_{c}^{2}$ (where the mandatory concepts are represented in bold) with:

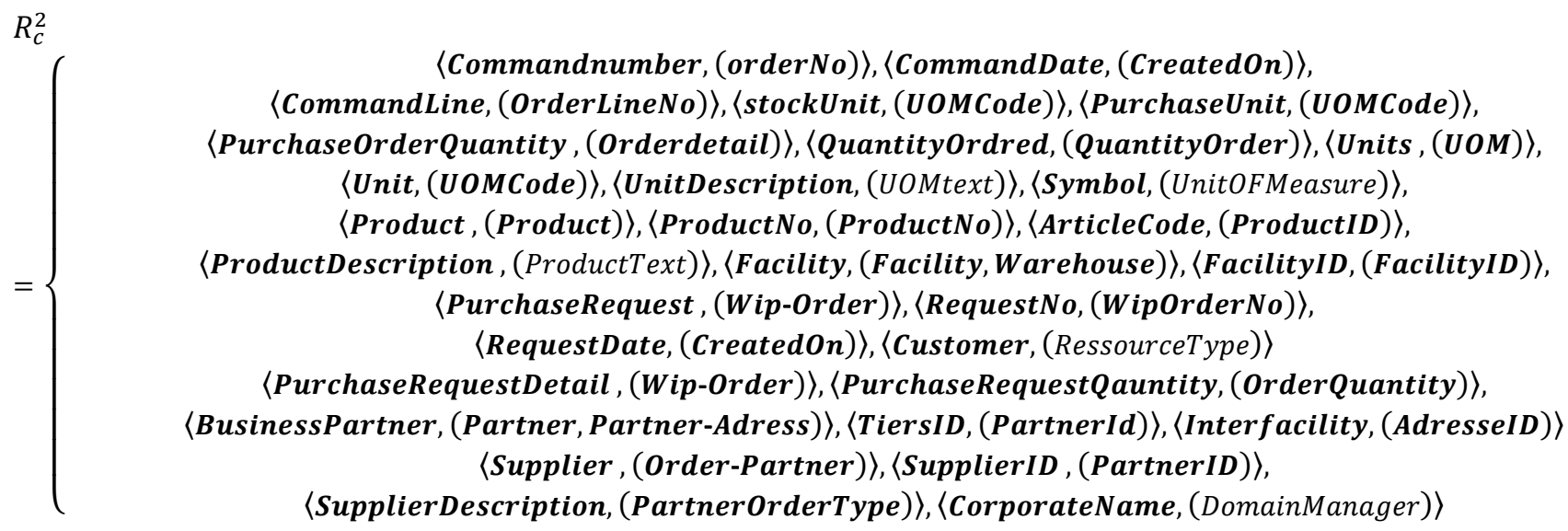


Moreover, we can identify $R_{c}^{2 e}$ by:

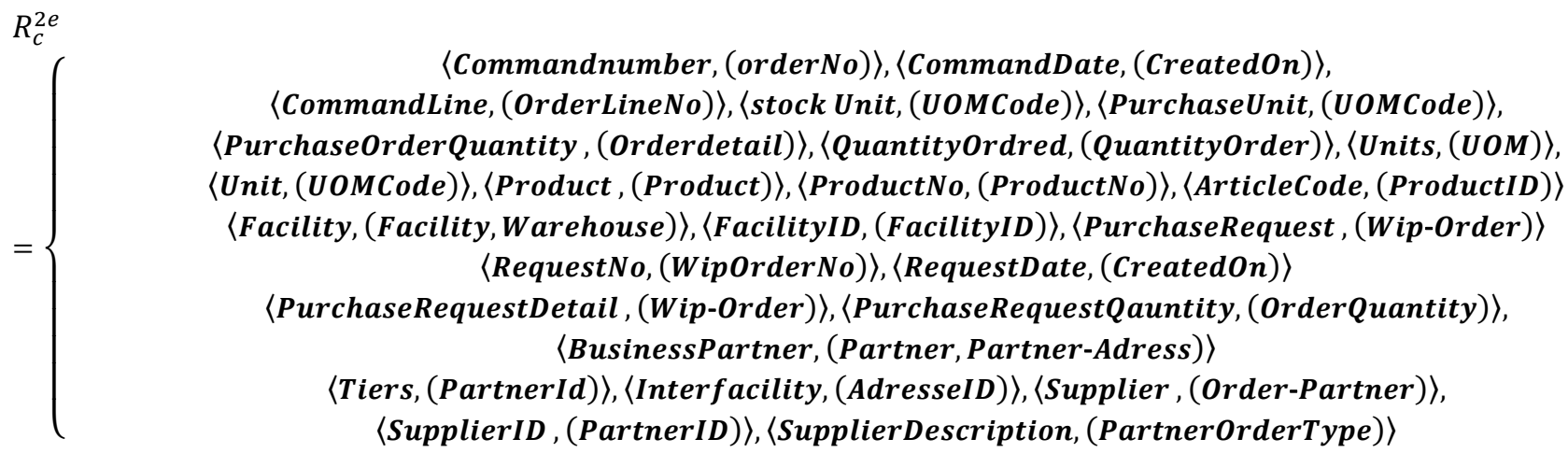

- The potential interoperability:

We have $\left|R_{c}^{2}\right|=30,\left|R_{c}^{2 e}\right|=25$ and $\left|R_{c_{\text {expected }}}^{2}\right|=\left|M C_{I S_{2}}\right|=37$

We could then calculate:

$$
\begin{gathered}
v_{1 \rightarrow 2}=\frac{\left|R_{c}^{2}\right|}{\mid R_{c_{\text {expected }}^{2} \mid}^{2}}=\frac{30}{37}=81 \% \\
\varepsilon_{1 \rightarrow 2}=\frac{\left|R_{c}^{2 e}\right|}{\left|R_{c}^{2}\right|}=\frac{25}{30}=83 \%
\end{gathered}
$$

The value $v_{1 \rightarrow 2}=86 \%<100 \%$ then the MES is partially interoperable with the ERP. Moreover, $\varepsilon_{1 \rightarrow 2}=83 \%$ of the semantic relationships given by $R_{c}^{2}$ are effective. Thus, the maximal potential interoperability level corresponds to the point $M P I_{1 \rightarrow 2}(81 \%, 83 \%)($ Figure 10).

Besides, the semantic gap is equal to $100 \%-v_{1 \rightarrow 2}=19 \%$, where, the missing semantics is given by the semantics of the concepts $c_{j}^{2}$ from $C_{I S_{2}}$ for which $R_{c_{j}}^{2}$ cannot be computed: these semantic relationships are identified in $R_{c_{\text {expected }}}^{2} \backslash R_{c}^{2}$. These concepts are:

Command Type, Purchase Order, Total Included Taxes, Country, Product - Facility, CreationDate,

PurchaseRequestLine. When analysing these missing concepts to ensure a fully potential interoperability, we could distinguish two mains concept categories based on the effort deployed to reduce the semantic gap. For instance, the first category contains the concepts that could easily be defined, i.e. CreationDate, Total Included Taxes, and Country. In fact, those concepts may be simply retrieved by considering for example the date of the day for the concept CreationDate. Whereas, the second category gathers all the concepts that may not be easily found, for example, PurchaseOrder and ProductFacility. In this case, experts are needed to proceed with the definition of the corresponding concepts for the second category. The experts' actions take part in the second improvement cited in section 5.3 .2 in order to reach the full interoperability.

The value $100 \%-\varepsilon_{1 \rightarrow 2}=17 \%$ represents the risk to retrieve non effective semantic relationships among $R_{c}^{2}$. Actually, five concepts taking part in semantic relationships are non-mandatory: (DomainManager, ResourceType, ProductText, 
UOMText, UnitOfMeasure).Thus, there is no guarantee that they are valued by the application's user and then they may be not exchanged during the interoperation process.

- The effective interoperability:

We compute:

$$
v_{1 \rightarrow 2}^{e}=\frac{\left|R_{c}^{2 e}\right|}{\left|R_{c_{\text {expected }}}^{2 e}\right|}=\frac{25}{37}=68 \%
$$

And,

$$
\varepsilon_{1 \rightarrow 2}^{e}=100 \%
$$

The value $v_{1 \rightarrow 2}^{e}=68 \%<100 \%$ then the MES is partially interoperable with the ERP but this partial interoperability is fully effective. Thus, the minimal effective interoperability level corresponds to the point $M E I_{1 \rightarrow 2}(71 \%, 100 \%)$ (Figure 10).

6.2. Interoperability evaluation from $\operatorname{ERP}\left(I S_{2}\right)$ to the MES $\left(I S_{1}\right)$

Let us now consider the evaluation of semantic interoperability from the ERP $\left(I S_{2}\right)$ to the MES $\left(I S_{1}\right)$.

The domain expert returns the semantic relationships $R_{c}^{1}$ and $R_{c}^{1 e}$ (where the mandatory concepts are written in bold)

$R_{C}^{1}$

$$
=\{
$$

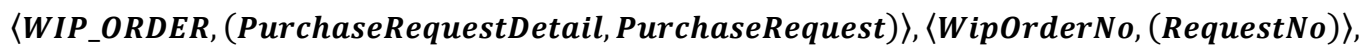
$\langle$ CreatedOn, (RequestDate) $\rangle,\langle$ OrderQuantity, (PurchaseOrder) $\rangle$,

$\langle$ ORDER - DETAIL, (PurchaseRequestQuantity) $\rangle,\langle$ OrderLineNo, (CommandLineNo) $\rangle$, $\langle$ ORDER - HEADER, (PurchaseOrder) $\rangle,\langle$ OrderDate, (CommandDate) $)$, $\langle$ OrderNo, (CommandNumber) $\rangle,\langle$ UOM, (Units) $\rangle,\langle$ UOMcode, (Unit) $\rangle$, $\langle$ FACILITY, (Facility) $\rangle,\langle$ FacilityID, (FacilityID) $)\rangle,\langle$ Division, (FacilityType $)\rangle$, $\langle$ PARTNER - ADRESS, (Facility) $\rangle,\langle$ AdressID, (FacilityID) $\rangle$,

$\langle$ PARTNER , (BusinessPartner, Supplier) $\rangle,\langle$ Partner - ID , (TiersID, SupplierID $)\rangle$, $\langle$ WarehouseID, (FacilityID) $\rangle,\langle$ Warehouse, (FACILITY, ProductFacility) $\rangle$,

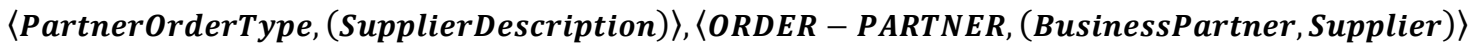
$\langle$ ORDER - ST ATUS, (PurchaseOrderStatus) $\rangle,\langle$ OrderStatus, (PurchaseOrderStatus) $\rangle$ $\langle$ PRODUCT, (Product) $\rangle,\langle$ ProductNo, (ProductNo $)\rangle,\langle$ ProductID, (ArticleCode $)\rangle$

And

$R_{c}^{1 e}$

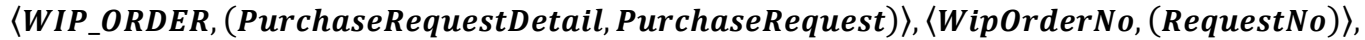
$\langle$ CreatedOn, (RequestDate) $\rangle,\langle$ OrderQuantity, (PurchaseOrder) $\rangle$,

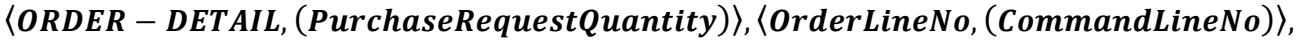
$\langle$ ORDER - HEADER, (PurchaseOrder) $\rangle,\langle$ OrderDate, (CommandDate) $)$, $\langle$ OrderNo, (CommandNumber) $\rangle,\langle$ UOM, (Units) $\rangle,\langle$ UOMcode, (Unit) $\rangle$, $\langle$ FACILITY, (Facility) $\rangle,\langle$ FacilityID, (FacilityID $)\rangle,\langle$ Division, (FacilityType $)\rangle$, $\langle$ PARTNER - ADRESS, (Facility) $\rangle,\langle$ AdressID, (FacilityID) $\rangle$,

$\langle$ PARTNER, (BusinessPartner, Supplier) $\rangle,\langle$ Partner - ID , (TiersID, SupplierID $)\rangle$, $\langle$ WarehouseID, (FacilityID) $\rangle,\langle$ Warehouse, (FACILITY, ProductFacility) $\rangle$,

$\langle$ PartnerOrderType, (SupplierDescription) $\rangle,\langle$ ORDER - PARTNER, (BusinessPartner, Supplier) $\rangle$ $\langle$ PRODUCT, (Product) $),\langle$ ProductNo, (ProductNo $)\rangle,\langle$ ProductID, (ArticleCode $)\rangle$ 
- The potential interoperability:

We have $\left|R_{c}^{1}\right|=27,\left|R_{c}^{1 e}\right|=25$ and $\left|R_{c_{\text {expected }}}^{1}\right|=40$

We could then calculate:

$$
\begin{gathered}
v_{2 \rightarrow 1}=\frac{\left|R_{c}^{1}\right|}{\left|R_{C_{\text {expected }}}^{1}\right|}=\frac{27}{40}=67.5 \% \\
\varepsilon_{2 \rightarrow 1}=\frac{\left|R_{c}^{1 e}\right|}{\left|R_{c}^{1}\right|}=\frac{25}{27}=92 \%
\end{gathered}
$$

The value $v_{2 \rightarrow 1}=67.5 \%<100 \%$ then the ERP is partially interoperable with the MES. Moreover, $\varepsilon_{2 \rightarrow 1}=92 \%$ of the semantic relationships given by $R_{c}^{1}$ are effective. Thus, the maximal potential interoperability level corresponds to the point $M P I_{2 \rightarrow 1}(67.5 \%, 92 \%)$ (Figure 10).

Besides, the semantic gap is equal to $100 \%-v_{2 \rightarrow 1}=32.5 \%$, where, the missing semantics is given by the semantics of the concepts $c_{j}^{1}$ from $C_{I S_{1}}$ for which $R_{c_{j}}^{1}$ cannot be computed: these semantic relationships are identified in $R_{c_{\text {expected }}}^{1} \backslash R_{c}^{1}$ and are WIP_ORDER_TYPE, WipOrderType, WIP_ORDER_STATUS, WipOrderStatus, CreatedOn, PartnerOrderNo, FUID, ProcessDescription, RevisionControlFlag, SerialTrackingCode, LotTrackingCode, PROCESS, ProcessId. This semantic gap could be characterised through two major categories depending on the effort deployed to bridge the gap. The first category gathers all the concepts that could be easily added to the information systems conceptual model, for instance the concept CreatedOn concerns the day date and could automatically be instantiated. The second category gathers the concepts whose semantics is hardly retrieved and added into the information systems conceptual model. This is the case for the concepts WIP_ORDER_STATUS and WIP_ORDER_TYPE as we could imagine that the information system users haven't the necessary knowledge to correctly instantiate them.

The value $100 \%-\varepsilon_{2 \rightarrow 1}=8 \%$ represents the risk to return non effective semantic relationships from $R_{c}^{1}$. This is due to some non-mandatory characteristic concepts participating in such semantic relationships.

\section{- The effective interoperability:}

We compute:

$$
v_{2 \rightarrow 1}^{e}=\frac{\left|R_{c}^{1 e}\right|}{\left|R_{\text {expected }}^{1}\right|}=\frac{25}{40}=62.5 \%
$$

And,

$$
\varepsilon_{2 \rightarrow 1}^{e}=100 \%
$$

The value $v_{2 \rightarrow 1}^{e}=62.5 \%<100 \%$ then the ERP is partially interoperable with the MES but this partial interoperability is fully effective. Thus, the minimal effective interoperability level corresponds to the point $M E I_{2 \rightarrow 1}(62.5 \%, 100 \%)$ (Figure 10). 


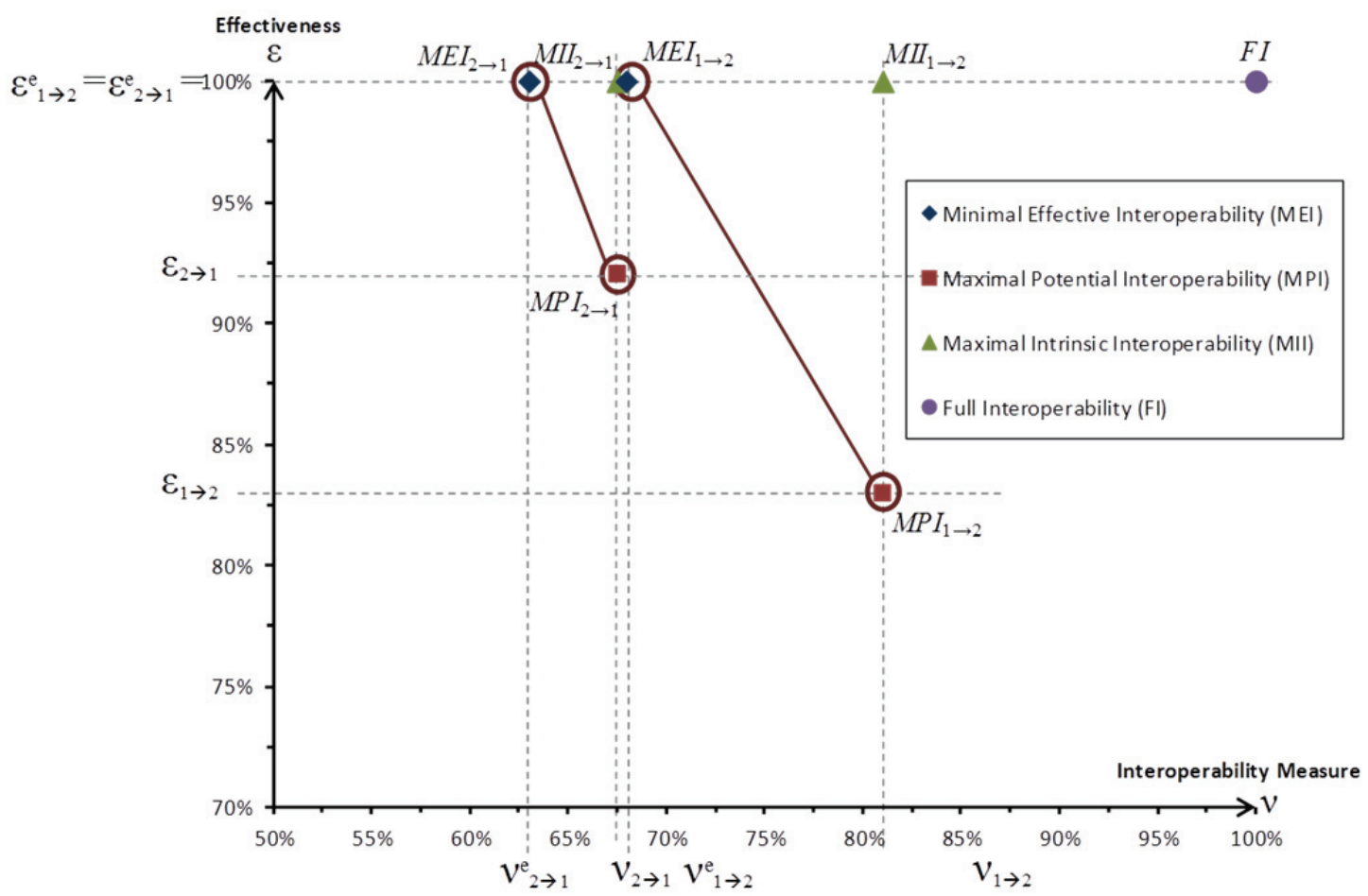

Figure 10.Interoperability measures map for both interoperability assessment directions

Taking into consideration the actual degree of interoperability between both information systems in both evaluation directions (see Figure 10), different actions could be conducted to improve these rates based on collaborative enterprises strategies. In this case, one action could be privileged over another one due to its cost and/or its time consumption. In the following section we define the eventual correlation between the different measures.

\subsection{Interoperability evaluation Improvements}

In this section, we only consider the improvement to reach the Maximal Intrinsic Interoperability (points $M I I_{1 \rightarrow 2}$ and $M I I_{2 \rightarrow 1}$ in Figure 10). This effort could be conducted by transforming all the non-mandatory concepts into mandatory ones. We estimate that computing the second improvements that consist in adding the semantics should only be conducted by the experts of both information systems and then out of scope of this section.

We elaborate the non-mandatory knowledge transformation for both interoperation ways separately in order to study the effect of this transformation on the already established measures.

\subsubsection{The maximal intrinsic interoperability $\left(v_{1 \rightarrow 2}, \varepsilon_{1 \rightarrow 2}^{e}\right)$}

We transform all the non-mandatory concepts from $I S_{I}$ (which are DomainManager, ResourceType, ProductText, UOMText, and UnitOfMeasure) in order to reach the maximal intrinsic interoperability $\left(v_{1 \rightarrow 2}, \varepsilon_{1 \rightarrow 2}^{e}\right)$ with 
$v_{1 \rightarrow 2}=\frac{\left|R_{c}^{2}\right|}{\left|R_{\text {expected }}^{2}\right|}=\frac{30}{37}=81 \%$ and $\varepsilon_{1 \rightarrow 2}^{e}=\frac{\left|R_{c}^{2 e}\right|}{\left|R_{c}^{2}\right|}=\frac{30}{30}=100 \%$

This transformation affects the interoperability evaluation from ERP $\left(I S_{2}\right)$ to the MES $\left(I S_{1}\right)$ as presented in the following.

We define below the new computed semantic relationships $R_{c}^{1 *}$ and $R_{c}^{1 e *}$ (where the added mandatory concepts are underlined):

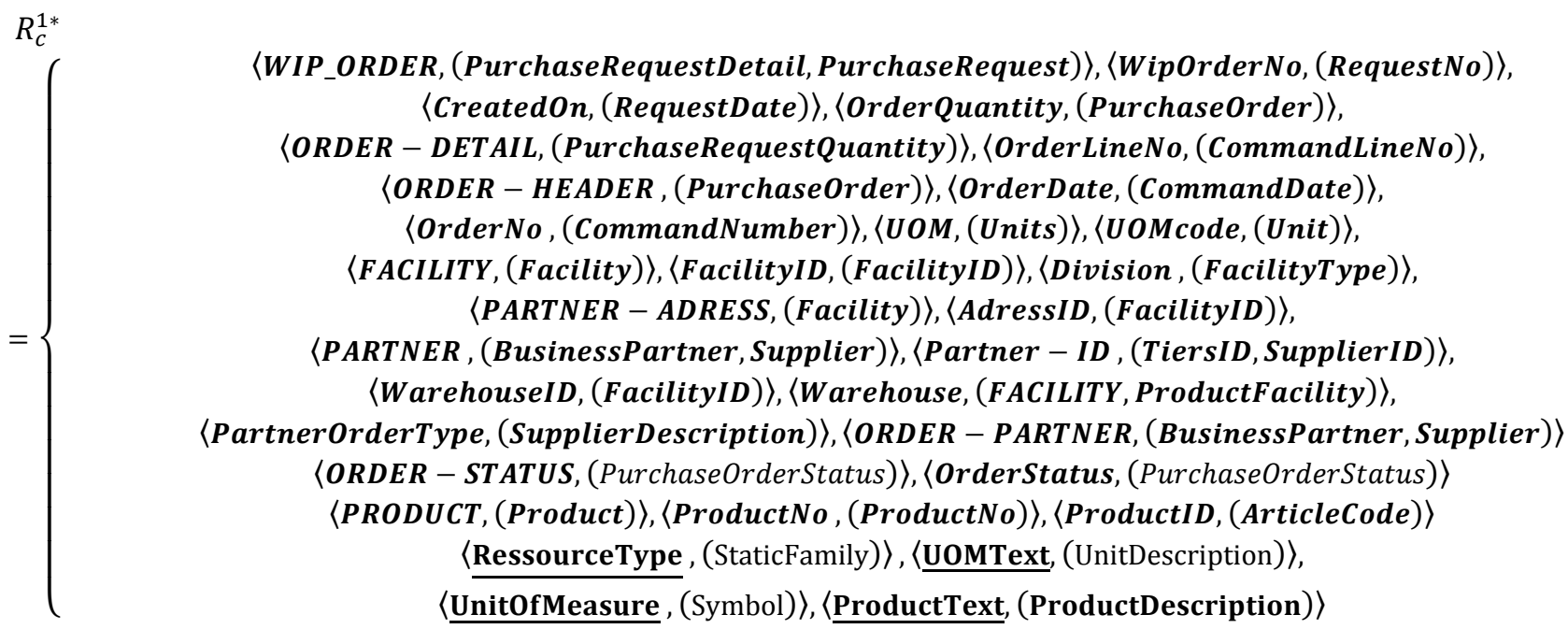

And

$R_{c}^{1 e *}$

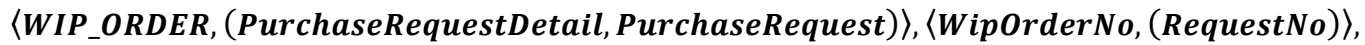

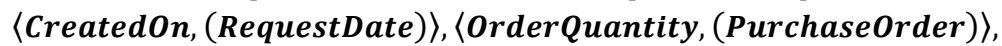

$\langle$ ORDER - DETAIL, (PurchaseRequestQuantity) $\rangle,\langle$ OrderLineNo, (CommandLineNo) $\rangle$, $\langle$ ORDER - HEADER, (PurchaseOrder) $\rangle,\langle$ OrderDate, (CommandDate $)\rangle$,

$\langle$ OrderNo, (CommandNumber) $\rangle,\langle$ UOM, (Units) $\rangle,\langle$ UOMcode, (Unit) $\rangle$,

$\langle$ FACILITY, (Facility) $\rangle,\langle$ FacilityID, (FacilityID $)\rangle,\langle$ Division, (FacilityType $)\rangle$, $\langle$ PARTNER - ADRESS, (Facility) $\rangle,\langle$ AdressID, (FacilityID $)\rangle$,

$\langle$ PARTNER , (BusinessPartner, Supplier) $\rangle,\langle$ Partner - ID , (TiersID, SupplierID $)\rangle$,

$\langle$ WarehouseID, (FacilityID) $\rangle,\langle$ Warehouse, (FACILITY, ProductFacility) $\rangle$,

$\langle$ PartnerOrderType, (SupplierDescription) $\rangle,\langle$ ORDER - PARTNER, (BusinessPartner, Supplier) $\rangle$

$\langle$ PRODUCT, (Product) $\rangle,\langle$ ProductNo, (ProductNo $)\rangle,\langle$ ProductID, (ArticleCode $)\rangle$

$\langle$ ProductText, (ProductDescription) $\rangle$

We find that $\left|R_{c}^{1 *}\right|=31,\left|R_{c}^{1 e^{* *}}\right|=26$ and $\left|R_{c_{\text {expected }}}^{1 *}\right|=45$

For the potential interoperability, we have:

$$
\begin{gathered}
v_{2 \rightarrow 1}^{*}=\frac{\left|R_{c}^{1 *}\right|}{\mid R_{C_{\text {expected }}^{1 *}}^{1 *}}=\frac{31}{45}=69 \% \\
\varepsilon_{2 \rightarrow 1}^{*}=\frac{\left|R_{c}^{1 e^{*}}\right|}{\left|R_{c}^{1 *}\right|}=\frac{26}{31}=84 \%
\end{gathered}
$$

For the effective interoperability, we have:

$$
v_{2 \rightarrow 1}^{e *}=\frac{\left|R_{c}^{1 e *}\right|}{\left|R_{C_{\text {expected }}^{1 *}}^{1 *}\right|}=\frac{26}{45}=58 \%
$$




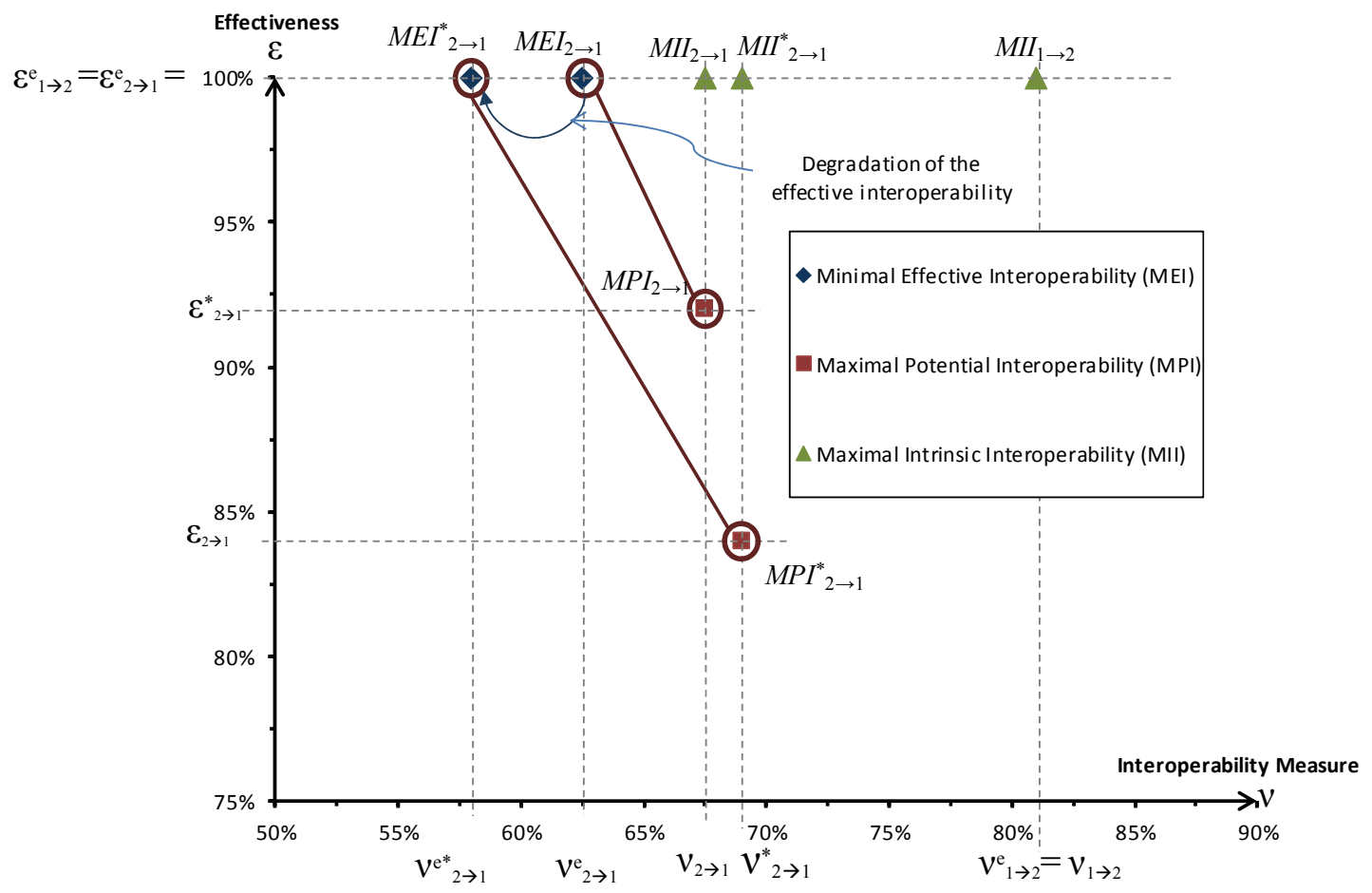

Figure 11.Degradation of the effective interoperability measure $v_{2 \rightarrow 1}^{e}$ after improving $v_{1 \rightarrow 2}^{e}$

6.3.2. The maximal intrinsic interoperability $\left(v_{2 \rightarrow 1}, \varepsilon_{2 \rightarrow 1}^{e}\right)$

We transform the entire non-mandatory concept from $I S_{2}$ which is (PurchaseOrderStatus) in order to reach the maximal intrinsic interoperability $\left(v_{2 \rightarrow 1}, \varepsilon_{2 \rightarrow 1}^{\mathrm{e}}\right)$ with $v_{2 \rightarrow 1}=\frac{\left|R_{c}^{1}\right|}{\left|R_{c_{\text {expected }}}\right|}=\frac{27}{40}=67.5 \%$ and $\varepsilon_{2 \rightarrow 1}^{e}=100 \%$.

This transformation affects the interoperability evaluation from the $\operatorname{MES}\left(I S_{1}\right)$ to the $\operatorname{ERP}\left(I S_{2}\right)$.

We define below the new computed semantic relationships $R_{c}^{2 *}$ and $R_{c}^{2 e *}$ (where the added mandatory concepts are underlined):

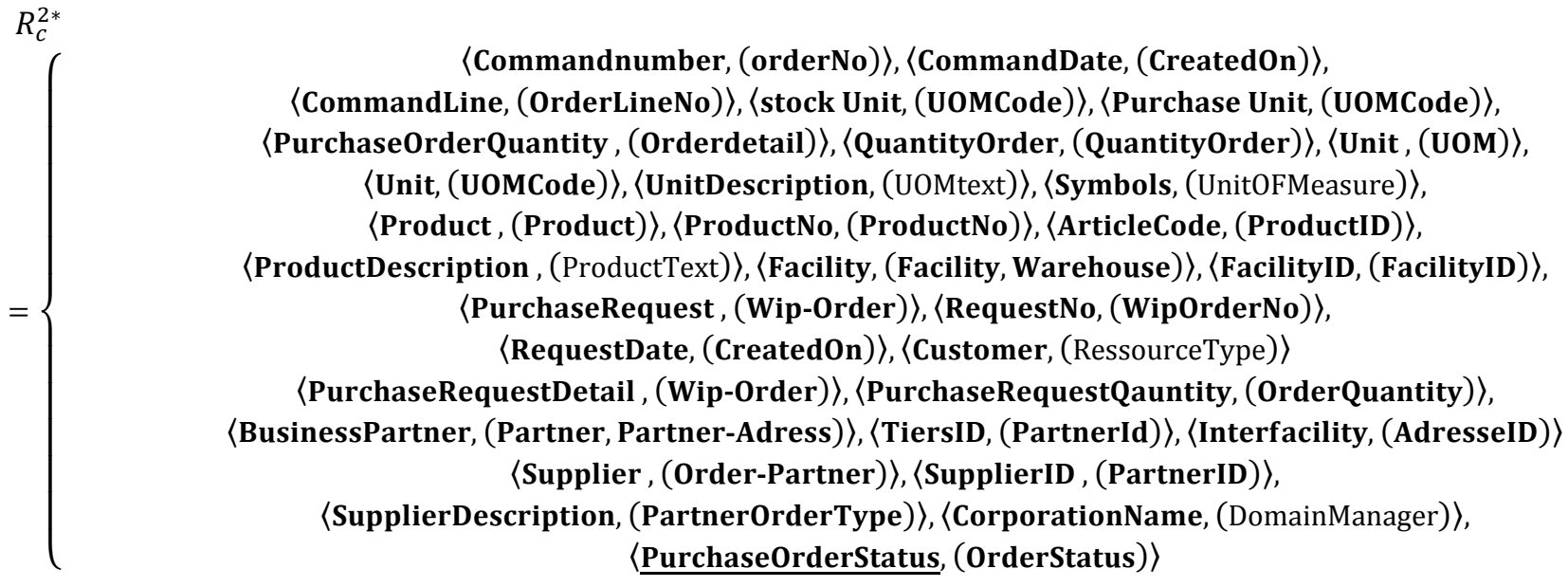

$\langle$ Commandnumber, (orderNo) $\rangle,\langle$ CommandDate, (CreatedOn) $)$,

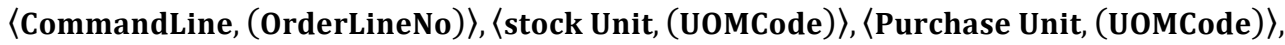
$\langle$ PurchaseOrderQuantity, (Orderdetail) $\rangle,\langle$ QuantityOrder, (QuantityOrder) $\rangle,\langle$ Unit , (UOM) $\rangle$, $\langle$ Unit, (UOMCode) $\rangle,\langle$ UnitDescription, (UOMtext) $\rangle,\langle$ Symbols, (UnitOFMeasure) $\rangle$, $\langle$ Product, (Product) $\rangle,\langle$ ProductNo, (ProductNo) $\rangle,\langle$ ArticleCode, (ProductID) $\rangle$,

$\langle$ ProductDescription, (ProductText) $\rangle,\langle$ Facility, (Facility, Warehouse) $\rangle,\langle$ FacilityID, (FacilityID) $\rangle$, $\langle$ PurchaseRequest, (Wip-Order) $\rangle$, 〈RequestNo, (WipOrderNo) $\rangle$, $\langle$ RequestDate, (CreatedOn) $\rangle,\langle$ Customer, (RessourceType) $\rangle$

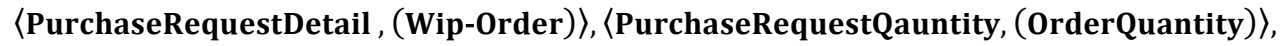
$\langle$ BusinessPartner, (Partner, Partner-Adress) $\rangle,\langle$ TiersID, (PartnerId) $\rangle,\langle$ Interfacility, (AdresseID) $\rangle$ $\langle$ Supplier , (Order-Partner) $\rangle$, 〈SupplierID , (PartnerID) $\rangle$,

$\langle$ SupplierDescription, (PartnerOrderType) $\rangle,\langle$ CorporationName, (DomainManager) $\rangle$, $\langle$ PurchaseOrderStatus, (OrderStatus) $\rangle$ 
$\langle$ Commandnumber, (orderNo) $\rangle,\langle$ CommandDate, (CreatedOn) $)$,

$\langle$ CommandLine, (OrderLineNo) $\rangle$, 〈stock Unit, (UOMCode) $\rangle,\langle$ Purchase Unit, (UOMCode) $\rangle$, $\langle$ PurchaseOrderQuantity, (Orderdetail) $\rangle,\langle$ QuantityOrder, (QuantityOrder) $\rangle,\langle$ Unit , (UOM) $\rangle$, $\langle$ Unit, (UOMCode) $\rangle$, 〈Product, (Product) $\rangle,\langle$ ProductNo, (ProductNo) $\rangle,\langle$ ArticleCode, (ProductID) $\rangle$ $\langle$ Facility, (Facility, Warehouse) $\rangle,\langle$ FacilityID, (FacilityID) $\rangle,\langle$ PurchaseRequest , (Wip-Order) $\rangle$ $\langle$ RequestNo, (WipOrderNo) $\rangle,\langle$ RequestDate, (CreatedOn) $\rangle$

$\langle$ PurchaseRequestDetail , (Wip-Order) ), 〈PurchaseRequestQauntity, (OrderQuantity)〉, $\langle$ BusinessPartner, (Partner, Partner-Adress) $\rangle$, 〈Tiers, (PartnerId) $\rangle$, $\langle$ Interfacility, (AdresseID) $\rangle,\langle$ Supplier , (Order-Partner) $\rangle,\langle$ SupplierID , (PartnerID) $\rangle$, $\langle$ SupplierDescription, (PartnerOrderType) $\rangle,\langle$ PurchaseOrderStatus, (OrderStatus) $\rangle$

We have $\left|R_{c}^{* 2}\right|=31,\left|R_{c}^{2 e *}\right|=26$ and $\left|R_{c_{\text {expected }}}^{1 *}\right|=38$

For the potential interoperability, we have:

For the effective interoperability, we have:

$$
\begin{gathered}
v_{1 \rightarrow 2}^{*}=\frac{\left|R_{c}^{2 *}\right|}{\mid R_{C_{\text {expected }}^{2 *} \mid}^{2 *}}=\frac{31}{38}=81.5 \% \\
\varepsilon_{1 \rightarrow 2}^{*}=\frac{\left|R_{c}^{22^{*}}\right|}{\left|R_{c}^{2 *}\right|}=\frac{26}{31}=84 \%
\end{gathered}
$$

$$
v_{1 \rightarrow 2}^{e *}=\frac{\left|R_{c}^{2 e *}\right|}{\left|R_{C_{\text {expected }}^{2 *} \mid}^{2}\right|}=\frac{26}{38}=68.4 \%
$$

The Figure 12 illustrates the different variations of the measures.

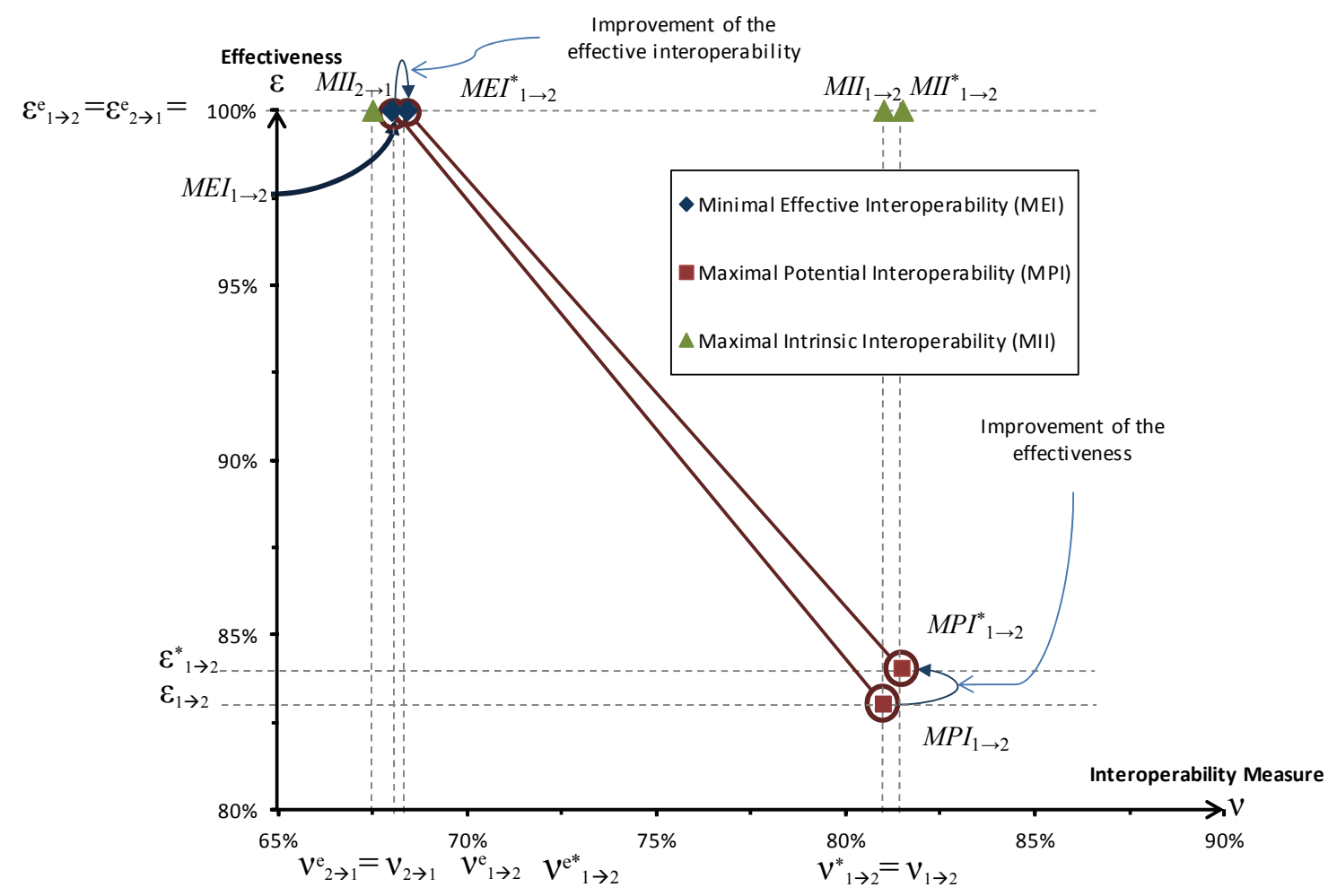

Figure 12.Effect on the interoperability evaluation from the MES $\left(I S_{1}\right)$ to the $\operatorname{ERP}\left(I S_{2}\right)$ 


\section{Conclusion and perspectives}

In distributed networks, autonomous and heterogeneous information systems lead up to cooperate in order to achieve common goals. This cooperation involves necessarily the capacity of those $I S$ s to interoperate. We proposed in this paper to evaluate the lack of interoperation through the measurement of the semantic gap occurring between the ISs conceptual models. To achieve this purpose, we first proposed to formalise the semantic relationships between $I S$ conceptual models. Based on those formal definitions, we formalised two measures, the potential and the effective interoperability, for assessing the interoperability between two ISs. An interoperability measures map facilitates, not only identifying the semantic gap, but also evaluating the actions to be taken in order to improve the effective interoperation.

The approach is illustrated on an extract of existing B2M applications: the ERP Sage X3 and the MES Flexnet which are cooperating in order to insure the manufacturing of a certain family of products.

The starting point of our formal approach is basically achieved by an expert of the domain and thus is a kind of limit when we are dealing with large scale CISs. Future work should provide a semi-automatic identification and formalisation of the semantic relationships by means of algorithms and tools developed by other research communities.

Moreover, we proposed a formal interoperability assessment approach that provides measures in order to assess quantitatively and qualitatively the interoperation between collaborative information systems. One of our perspectives is to conjugate our formal approach with different maturity models [5] that are mainly based on semi-formal evaluation.

Besides, we have defined the various actions that could be conducted by the collaborative enterprises in order to improve its potential and effective interoperability. Future works should detail how this action could be conducted, i.e. how to add the mandatory knowledge when studying a given way of interoperation and what could be the effect on that transformation on the interoperation in the other way? We are studying the possible correlation between both interoperation ways.

Another main perspective concerns the application of our approach and measures in the domain of Systems Engineering [48], and mainly in the formation of Systems-of-Systems [4] for evaluating the needed actions for these systems to be setup.

\section{References}

[1] L. Whitman, D. Santanu, and H. Panetto, An enterprise model of interoperability, in Proceeding of the 12th IFAC Symposium on Information Control Problems in Manufacturing, vol. 12, Saint Etienne,France, IFAC-PapersOnline, 2006.

[2] D.A. Fisher, An emergent perspective on interoperation in systems of systems, Carnegie-Mellon University of Pittsburgh, , Software Engineering Institute, Technical Report CMU/SEI-2006-Tr-003, ESC-Tr-2006-003, 2006.

[3] IEEE, Standard Computer Dictionary - A Compilation of IEEE Standard Computer Glossaries, 1990.

[4] D. Carney, D. Fisher, and P. Place, Topics in Interoperability: System-of-Systems Evolution, University of Pittsburgh, Software Engineering Institute, Technical Note CMU/SEI-2005-TN-002, 2005. 
[5] H. Panetto, Towards a classification framework for interoperability of enterprise applications, International Journal of Computer Integrated Manufacturing, vol. 20, no. 8, Taylor \& Francis, pp. 727-740, December (2007).

[6] EIF, European Interoperability Framework for pan-European eGovernment Services,Interoperable Delivery of European eGovernment Services to public Administrations,Businesses and Citizens (IDABC), 2004.

[7] D. Chen; M. Dassisti;B. Elvesaeter; H. Panetto; N. Daclin; F-W. Jaekel; T. Knothe; A. Solberg; V. Anaya; R. Sanchis, et al., Enterprise Interoperability-Framework and Knowledge corpus, INTEROP-NOE FP6 IST-2003-508011, Deliverable DI.2, http://www.interop-vlab.eu, 2006.

[8] C.M.Chituc, C.Toscano, and A.Azevedo. Interoperability in Collaborative Networks: Independent and industryspecific initiatives-The case of the footwear industry. Computers in Industry, vol59, no 7,pp 741-757(2008).

[9] H. Panetto. andA. Molina. Enterprise integration and interoperability in manufacturing systems: Trends and issues. Computers in industry, vol59,no 7, pp641-646 (2008).

[10] Interoperability Working group C4ISR, Levels of information systems interoperability (LISI), US Department of defense, Washington, DC, Architectures Working Group report February 1998.

[11] T. Clark and R. Jones, Organisational interoperability maturity model for $\mathrm{C} 2$, in Proceedings of the Command and Control Research and Technology Symposium (CCRTS), Newport, RI, USA, 1999.

[12] A. Tolk, Beyond technical interoperability-Introducing a reference model for measures of merit for coalition interoperability, in Proceedings of the 8th International Command and Control Research and Technology Symposium (ICCRTS), Washington, DC, June 17-19, 2003.

[13] ISOFDIS 11354-1, Advanced automation technologies and their applications-Requirements for establishing manufacturing enterprise process interoperability - part 1: framework for enterprise interoperability, ISO, Geneva, Switzerland, TC 184/SC 52010.

[14] D. Chen and N. Daclin, Barriers driven methodology for enterprise interoperability, in Establishing the foundation of collaborative networks. IFIP TC 5 WG 5.5, in Proceedings of $8^{\text {th }}$ IFIP Working Conference on Virtual Enterprises, vol. 243, Guimaraes, Portugal, September 10-12, 2007, pp. 453-460.

[15] W. Guédria, Y. Naudet, and D. Chen, Interoperability Maturity Models--Survey and Comparison, in Proceedings of $3^{\text {th }}$ International workshops on Enterprise, Interoperability and Networking (EI2N 2008). OTM Confederated International Workshops and Posters, Monterrey, Mexico, LNCS 5333, Springer, pp. 273-282, November 2008.

[16] T. Ford, J. Colombi, S. Graham, and D. Jacques, The Interoperability Score, in Proceeding of the 5th Conference on Systems Engineering Research, Hoboken, NJ, USA, March 14-16, 2007.

[17] N. Daclin, D. Chen, and B. Vallespir, Methodology for enterprise interoperability, in Proceedings of the 17th World Congress: The International Federation of Automatic Control, Seoul, Korea, July 2008, pp. 6-11.

[18] S. Castano, and V. DeAntonellis, A framework for expressing semantic relationships between multiple information systems for cooperation.Information Systems, vol. 23, no. 3-4, pp. 253-277, (1998).

[19] D.Bianchini, V. De Antonellis, M.Melchiori, and D. Salvi, Semantic-enriched service discovery,in Proceedings of the 22nd International Conference on Data Engineering WorkshopsICDEW '06. IEEE Computer Society, Atlanta, GA, USA, April 2006, pp.38-48.

[20] C. Cleverdon. The Cranfield tests on index language devices. In MCB UP Ltd, 173-194 (1993).

[21] H.H. Do, S. Melnikand E. Rahm.Comparison of schema matching evaluation.Web, Web-Services, and Database Systems, pp 221-237 (2009).

[22] M. Ehrig and J. Euzenat. Relaxed precision and recall for ontology matching. In Proceedings of Integrating Ontologies Workshop, Banff, Canada, 2005 
[23] E. Yahia, M. Lezoche, A. Aubry, and H. Panetto, Semantics enactment in Enterprise Information Systems, in Proceedings of the 18th World Congress: The International Federation of Automatic Control, Milan, Italy, August 28September 4, 2011.

[24] T. Halpin, Fact-oriented modelling: Past, present and future, Conceptual Modelling in Information Systems Engineering, pp. 19-38, (2007).

[25] T. Halpin, Object-role modeling (ORM/NIAM), Handbook on Architectures of Information Systems, pp. 81-103, 2006.

[26] B. Aleman-Meza, C. Halaschek, I.B. Arpinar, and A. Sheth, Context-aware semantic association ranking, in Proceedings of Semantic Web and Databases,Berlin, Germany, vol. 3, June 2003, pp. 33-50.

[27] A. Sheth; B. Aleman-Meza; I.B. Arpinar; C. Bertram; Y. Warke; C. Ramakrishanan; C. Halaschek; K. Anyanwu; D. Avant; F-S. Arpinar; et al., Semantic association identification and knowledge discovery for national security applications, Journal of Database Management, vol. 16, no. 1, pp. 33-53, (2005).

[28] N. Choi, I.Y. Song, and H. Han, A survey on ontology mapping, ACM Sigmod Record, vol. 35, no. 3, pp. 34-41, (2006).

[29] E. Rahm and P.A. Bernstein, A survey of approaches to automatic schema matching, the VLDB Journal, vol. 10, no. 4, pp. 334-350, (2001).

[30] J. Evermann, Theories of meaning in schema matching: An exploratory study. Information Systems, vol. 34, no.1, pp. 28-44, (2009).

[31] G. Wang, J. Goguen, Y.K. Nam, and K. Lin, Critical points for interactive schema matching, in Proceedings of 6th Asia-Pacific Web Conference: Advanced Web Technologies and Applications, APweb 2004, Hangzhou, China, April 14-17, pp. 654-664, 2004.

[32] J. Goguen and R. Burstall, Introducing institutions, in Proceedings of the Carnegie Mellon Workshop on Logic of Programs, Pittsburgh, PA, USA, LNCS 164, Springer, pp. 221-256, June 6-8, 1983.

[33] A. Isaac; S. Wang; C. Zinn; H. Matthezing; L. van. der ; S. Schlobach, Evaluating thesaurus alignments for semantic interoperability in the library domain, IEEE Intelligent Systems, vol. 24, no. 2, pp. 76-86, (2009).

[34] J.J. Jung, Reusing ontology mappings for query routing in semantic peer-to-peer environment, Information Sciences, vol. 180, no. 17, pp. 3248-3257, (2010).

[35] J. David and J. Euzenat, Comparison between ontology distances (preliminary results), in Proceedings of the $7^{\text {th }}$ International Semantic Web Conference, ISWC 2008: Karlsruhe, Germany, October 2008, pp. 245-260.

[36] C.P. Cheng, G.T. Lau, J. Pan, K.H. Law, and A. Jones, Domain-specific ontology mapping by corpus-based semantic similarity, in Proceedings of 2008 NSF CMMI Engineering Research and Innovation Conference, Knoxville, TN, USA, January 2008.

[37] A. Shilliday, J. Taylor, M. Clark, and S. Bringsjord, Provability-Based Semantic Interoperability for Information Sharing and Joint Reasoning, in Proceeding of the 2010 conference on Ontologies and Semantic Technologies for Intelligence,Frontiers in Artificial Intelligence and Applications, 213, IOS Press, 2010, pp. 109-128.

[38] J.A. Goguen, Data, schema, ontology and logic integration, Logic Journal of IGPL, vol. 13, no. 6, pp. 685-715, (2005).

[39] Y. Kalfoglou and M. Schorlemmer, Ontology mapping: the state of the art, The knowledge engineering review, vol. 18, no. 1, pp. 1-31, (2003).

[40] E. Yahia, J. Yang, A. Aubry, and H. Panetto, On the Use of Description Logic for Semantic Interoperability of Enterprise Systems, in Proceedings of $4^{\text {th }}$ International workshops on Enterprise, Interoperability and Networking (EI2N 
2009). OTM Confederated International Workshops and Posters, Villamoura, Portugal, LNCS 5872, Springer, 2009, pp. 205215.

[41] C. Masolo, S. Borgo, A. Gangemi, N. Guarino, and A. Oltramari, WonderWeb Deliverable D18--Ontology Library (final), ST Project 2001-33052 WonderWeb: Ontology Infrastructure for the Semantic Web, Deliverable 18, http://www.wonderweb.semanticweb.org, 2003.

[42] S.L. Reed and D.B. Lenat, Mapping ontologies into Cyc, in Proceedings of AAAI 2002 Conference Workshop on Ontologies For The Semantic Web, Edmonton, Canada, pp. 1-6, 2002.

[43] D. M. Giménez, M. Vegetti, H. P. Leone, and G. P. Henning, PRoductONTOlogy: Defining product-related concepts for logistics planning activities, Computers in Industry, Elsevier, 59, 2-3, pp 231-241, March (2008).

[44] A. Tursi, H. Panetto, G. Morel, and M. Dassisti, Ontological approach for products-centric information system interoperability in networked manufacturing enterprises, IFAC Annual Reviews in Control, vol. 33, no. 3, pp. 238-245, (2009).

[45] F. Ruskey and M. Weston, A survey of Venn diagrams, Electronic Journal of Combinatorics, vol. 4, (1997).

[46] K.Q. He, J. Wang, and P. Liang, Semantic Interoperability Aggregation in Service Requirements Refinement, Journal of Computer Science and Technology, vol. 25, no. 6, pp. 1103-1117, (2010).

[47] V. Stroetmann, JM Rodrigues, and K. Stroetmann, Conceptual Framework for eHealth Interoperability, Deliverable 1.1 of the SemanticHEALTH Project. http://www.semantichealth.org, 2006.

[48] C. Haskins (Ed), INCOSE Systems Engineering Handbook: A Guide for Life Cycle Processes and Activities, Version 3,The International Council on Systems Engineering, 2006. 